\title{
THE BREZIS-NIRENBERG RESULT FOR THE FRACTIONAL LAPLACIAN
}

\author{
RAFFAELLA SERVADEI AND ENRICO VALDINOCI
}

Abstract. The aim of this paper is to deal with the non-local fractional counterpart of the Laplace equation involving critical non-linearities studied in the famous paper of Brezis and Nirenberg (1983). Namely, our model is the equation

$$
\begin{cases}(-\Delta)^{s} u-\lambda u=|u|^{2^{*}-2} u & \text { in } \Omega, \\ u=0 & \text { in } \mathbb{R}^{n} \backslash \Omega,\end{cases}
$$

where $(-\Delta)^{s}$ is the fractional Laplace operator, $s \in(0,1), \Omega$ is an open bounded set of $\mathbb{R}^{n}, n>2 s$, with Lipschitz boundary, $\lambda>0$ is a real parameter and $2^{*}=2 n /(n-2 s)$ is a fractional critical Sobolev exponent.

In this paper we first study the problem in a general framework; indeed we consider the equation

$$
\begin{cases}\mathcal{L}_{K} u+\lambda u+|u|^{2^{*}-2} u+f(x, u)=0 & \text { in } \Omega, \\ u=0 & \text { in } \mathbb{R}^{n} \backslash \Omega,\end{cases}
$$

where $\mathcal{L}_{K}$ is a general non-local integrodifferential operator of order $s$ and $f$ is a lower order perturbation of the critical power $|u|^{2^{*}-2} u$. In this setting we prove an existence result through variational techniques.

Then, as a concrete example, we derive a Brezis-Nirenberg type result for our model equation; that is, we show that if $\lambda_{1, s}$ is the first eigenvalue of the non-local operator $(-\Delta)^{s}$ with homogeneous Dirichlet boundary datum, then for any $\lambda \in\left(0, \lambda_{1, s}\right)$ there exists a non-trivial solution of the above model equation, provided $n \geqslant 4 s$. In this sense the present work may be seen as the extension of the classical Brezis-Nirenberg result to the case of non-local fractional operators.

\section{Contents}

1. Introduction

2. Some preliminary results

3. A general critical integrodifferential equation

4. A critical fractional Laplace equation

References

Received by the editors December 16, 2011 and, in revised form, May 29, 2012.

2010 Mathematics Subject Classification. Primary 49J35, 35A15, 35S15; Secondary 47G20, $45 \mathrm{G} 05$.

Key words and phrases. Mountain Pass Theorem, critical non-linearities, best critical Sobolev constant, variational techniques, integrodifferential operators, fractional Laplacian.

The first author was supported by the MIUR National Research Project Variational and Topological Methods in the Study of Nonlinear Phenomena, and the second author by the ERC grant $\epsilon$ (Elliptic Pde's and Symmetry of Interfaces and Layers for Odd Nonlinearities) and the FIRB project A\&B (Analysis and Beyond). 


\section{INTRODUCTION}

1.1. Fractional Laplace equations with critical non-linearities. Recently, a great deal of attention has been devoted to fractional and non-local operators of elliptic type, both for their interesting theoretical structure and in view of concrete applications (for several motivations, an elementary introduction to this topic and a - still not exhaustive - list of references see, e.g., 9]). Motivated by the interest shared by the mathematical community in this topic, we consider here the non-local counterpart of the following non-linear elliptic partial differential critical equation:

$$
\begin{cases}-\Delta u-\lambda u=|u|^{2_{*}-2} u & \text { in } \Omega, \\ u=0 & \text { on } \partial \Omega, \quad 2 *=2 n /(n-2), n>2,\end{cases}
$$

namely the non-local fractional equation

$$
\begin{cases}(-\Delta)^{s} u-\lambda u=|u|^{2^{*}-2} u & \text { in } \Omega, \\ u=0 & \text { in } \mathbb{R}^{n} \backslash \Omega,\end{cases}
$$

where $s \in(0,1)$ is fixed and $(-\Delta)^{s}$ is the fractional Laplace operator, which (up to normalization factors) may be defined as

$$
-(-\Delta)^{s} u(x)=\int_{\mathbb{R}^{n}} \frac{u(x+y)+u(x-y)-2 u(x)}{|y|^{n+2 s}} d y, \quad x \in \mathbb{R}^{n}
$$

(see for instance [9] and the references therein for further details on the fractional Laplacian), $\Omega \subset \mathbb{R}^{n}, n>2 s$, is open, bounded and with Lipschitz boundary, $\lambda>0$ and

$$
2^{*}=\frac{2 n}{n-2 s}
$$

is the fractional critical Sobolev exponent 1 The homogeneous Dirichlet datum in (1.2) is given in $\mathbb{R}^{n} \backslash \Omega$ and not simply on $\partial \Omega$, as it happens in (1.1), consistent with the non-local character of the operator $(-\Delta)^{s}$.

In the case when the non-linear term in problems like (1.1) has a subcritical growth (i.e., for instance, is of the form $|u|^{q-2} u$, with $q \in\left(2,2_{*}\right)$ ), the existence of solutions was investigated by many authors (see [16, 18, and the references therein), while the fractional analogue of these results for problem (1.2) can be found in [14, 15.

The aim of this paper is to investigate the critical case. In the classical critical setting in the famous paper 3. Brezis and Nirenberg proved that

a) if $n \geqslant 4$, then for any $\lambda \in\left(0, \lambda_{1}(-\Delta)\right)$ problem (1.1) has a positive solution;

b) if $n=3$, then there exists a constant $\lambda_{*} \in\left(0, \lambda_{1}(-\Delta)\right)$ such that for any $\lambda \in\left(\lambda_{*}, \lambda_{1}(-\Delta)\right)$ problem (1.1) has a positive solution.

In the case when $\Omega$ is a ball in $b$ ) they calculated explicitly $\lambda_{*}$ and proved that the following result (stronger than $b$ )) holds true:

c) when $n=3$ and $\Omega$ is a ball, problem (1.1) has a positive solution if and only if $\lambda \in\left(\lambda_{1}(-\Delta) / 4, \lambda_{1}(-\Delta)\right)$.

${ }^{1}$ We remark that

$$
2_{*}=\frac{2 n}{n-2}>\frac{2 n}{n-2 s}=2^{*}>2 .
$$

In our framework, the exponent $2^{*}$ plays the role of a fractional critical Sobolev exponent (see, e.g. [9], Theorem 6.5]). 
Here $\lambda_{1}(-\Delta)$ is the first eigenvalue of the Laplacian with homogeneous Dirichlet boundary conditions. In [5] Capozzi, Fortunato and Palmieri extended the existence result of [3], proving that if $n \geqslant 4$, then problem (1.1) has a solution for any $\lambda>0$.

Our aim is to show that the results of [3] (part a)) can be extended to problem (1.2). In order to do this, as in the classical case, we will use a variational technique: we can approach the problem at least in two different ways. The first one consists of noting that the solutions of (1.2) correspond to critical points of the 'free' functional defined as follows:

$$
u \mapsto \frac{1}{2} \int_{\mathbb{R}^{2 n}} \frac{|u(x)-u(y)|^{2}}{|x-y|^{n+2 s}} d x d y-\frac{\lambda}{2} \int_{\Omega}|u(x)|^{2} d x-\frac{1}{2^{*}} \int_{\Omega}|u(x)|^{2^{*}} d x .
$$

The second variational approach consists of looking for critical points of the functional

$$
u \mapsto \int_{\mathbb{R}^{2 n}} \frac{|u(x)-u(y)|^{2}}{|x-y|^{n+2 s}} d x d y-\lambda \int_{\Omega}|u(x)|^{2} d x
$$

on the sphere $\left\{u:\|u\|_{L^{2^{*}}(\Omega)}=1\right\}$. This second approach is related to the existence of the best fractional critical Sobolev constant.

In the model case (1.2) these two approaches are equivalent. On the other hand, for more general non-linearities with critical growth this is not true, since it is not always possible to reduce a boundary value problem to a constrained minimization problem: in this case, the use of the 'free' functional becomes, therefore, somewhat unavoidable.

In the present paper we first study the general integrodifferential equation

$$
\begin{cases}\mathcal{L}_{K} u+\lambda u+|u|^{2^{*}-2} u+f(x, u)=0 & \text { in } \Omega, \\ u=0 & \text { in } \mathbb{R}^{n} \backslash \Omega,\end{cases}
$$

where $s \in(0,1)$ is fixed, $\Omega \subset \mathbb{R}^{n}, n>2 s$, is open, bounded and with Lipschitz boundary, $\lambda>0$, and $\mathcal{L}_{K}$ is the non-local operator defined as follows:

$$
\mathcal{L}_{K} u(x)=\int_{\mathbb{R}^{n}}(u(x+y)+u(x-y)-2 u(x)) K(y) d y, \quad x \in \mathbb{R}^{n} .
$$

Here $K: \mathbb{R}^{n} \backslash\{0\} \rightarrow(0,+\infty)$ is a function such that

$$
m K \in L^{1}\left(\mathbb{R}^{n}\right) \text {, where } m(x)=\min \left\{|x|^{2}, 1\right\} ;
$$

there exists $\theta>0$ such that $K(x) \geqslant \theta|x|^{-(n+2 s)}$ for any $x \in \mathbb{R}^{n} \backslash\{0\}$;

$$
K(x)=K(-x) \text { for any } x \in \mathbb{R}^{n} \backslash\{0\} .
$$

A model for $K$ is given by $K(x)=|x|^{-(n+2 s)}$ and 2 in this case, $\mathcal{L}_{K}$ is - up to a normalization constant - the fractional Laplace operator $-(-\Delta)^{s}$ defined in (1.3).

\footnotetext{
${ }^{2}$ We think it is quite interesting to consider more general operators than simply the fractional Laplacian, since, in the applications, other types of non-local operators naturally arise (see, e.g., [8]). For instance, for the type of operators we consider here, it is not known if the problem can be reduced to a (possibly singular or degenerate) local one by the extension of 4 . We also think it is an interesting open problem to give concrete examples of fractional operators (other than the fractional Laplacian) for which conditions (1.25) and (1.27) may be checked explicitly. On the other hand, an advantage of our general approach is to adapt the variational techniques at once to all the operators with kernel $K$, by dealing with the appropriate functional spaces.
} 
The non-linear term in equation (1.5) is a Carathéodory function $f: \Omega \times \mathbb{R} \rightarrow \mathbb{R}$ verifying the following conditions:

$$
\begin{gathered}
\sup \{|f(x, t)|: \text { a.e. } x \in \Omega,|t| \leqslant M\}<+\infty \text { for any } M>0 ; \\
\lim _{t \rightarrow 0} \frac{f(x, t)}{t}=0 \text { uniformly in } x \in \Omega \\
\lim _{|t| \rightarrow+\infty} \frac{f(x, t)}{|t|^{2^{*}-1}}=0 \text { uniformly in } x \in \Omega .
\end{gathered}
$$

The term $f$ represents a lower order perturbation of the critical non-linearity $|u|^{2^{*}-2} u$. As a model for $f$ we can take the non-linearity $f(x, t)=a(x)|t|^{q}$, with $a \in L^{\infty}(\Omega)$ and $q \in\left(1,2^{*}-1\right)$.

In particular we are interested in the weak formulation of (1.5) given by the following problem (for this, it is convenient to assume (1.9) $)^{3}$

$$
\left\{\begin{array}{l}
\int_{\mathbb{R}^{2 n}}(u(x)-u(y))(\varphi(x)-\varphi(y)) K(x-y) d x d y-\lambda \int_{\Omega} u(x) \varphi(x) d x \\
\quad=\int_{\Omega}|u(x)|^{2^{*}-2} u(x) \varphi(x) d x+\int_{\Omega} f(x, u(x)) \varphi(x) d x \quad \forall \varphi \in X_{0}, \\
u \in X_{0} .
\end{array}\right.
$$

Here the functional space $X$ denotes the linear space of Lebesgue measurable functions from $\mathbb{R}^{n}$ to $\mathbb{R}$ such that the restriction to $\Omega$ of any function $g$ in $X$ belongs to $L^{2}(\Omega)$ and

the map $(x, y) \mapsto(g(x)-g(y)) \sqrt{K(x-y)}$ is in $L^{2}\left(\mathbb{R}^{2 n} \backslash(\mathcal{C} \Omega \times \mathcal{C} \Omega), d x d y\right)$, where $\mathcal{C} \Omega:=\mathbb{R}^{n} \backslash \Omega$, while

$$
X_{0}=\left\{g \in X: g=0 \text { a.e. in } \mathbb{R}^{n} \backslash \Omega\right\} .
$$

We note that

$$
C_{0}^{2}(\Omega) \subseteq X_{0}
$$

see, e.g., [13, Lemma 5.1] (for this we need condition (1.7)). Thus so $X$ and $X_{0}$ are non-empty.

1.2. Main results of the paper. Assumption (1.11) implies that $f(x, 0)=0$, so that the function $u \equiv 0$ is a (trivial) solution of (1.5). The aim of this paper will be, then, to find non-trivial solutions for (1.5). For this, we will adapt the variational techniques of [3] to the non-local setting. More precisely, we will study the critical points of the functional $\mathcal{J}_{K, \lambda}: X_{0} \rightarrow \mathbb{R}$ defined as follows:

$$
\begin{aligned}
\mathcal{J}_{K, \lambda}(u)=\frac{1}{2} & \int_{\mathbb{R}^{2 n}}|u(x)-u(y)|^{2} K(x-y) d x d y-\frac{\lambda}{2} \int_{\Omega}|u(x)|^{2} d x \\
& -\frac{1}{2^{*}} \int_{\Omega}|u(x)|^{2^{*}} d x-\int_{\Omega} F(x, u(x)) d x,
\end{aligned}
$$

\footnotetext{
${ }^{3}$ Condition (1.9) was assumed here only for the sake of simplicity and it can be easily removed. For instance, we do not need condition (1.9) in order to write (1.13). Indeed, [13, Lemma 5.6] holds true also without condition (1.9) (along its proof it is enough to consider a different change of variables in formula (5.18)).
} 
where $F$ is given by

$$
F(x, t)=\int_{0}^{t} f(x, \tau) d \tau .
$$

As in the classical case of the Laplacian, in the non-local setting the issue that needs to be overcome is that the functional $\mathcal{J}_{K, \lambda}$ does not satisfy the PalaisSmale condition. This lack of compactness is due to the fact that the embedding $X_{0} \hookrightarrow L^{2^{*}}\left(\mathbb{R}^{n}\right)$ (or, in the case of the fractional Laplacian, $H^{s}\left(\mathbb{R}^{n}\right) \hookrightarrow L^{2^{*}}\left(\mathbb{R}^{n}\right)$ ) is not compact. For this the functional $\mathcal{J}_{K, \lambda}$ does not verify the Palais-Smale condition globally, but - as we will see in the sequel - only in a suitable range related to the best fractional critical Sobolev constant in the embedding $X_{0} \hookrightarrow L^{2^{*}}\left(\mathbb{R}^{n}\right)$. For this, it is convenient to define

$$
S_{K}:=\inf _{v \in X_{0} \backslash\{0\}} S_{K}(v)
$$

where for any $v \in X_{0} \backslash\{0\}$

$$
\begin{aligned}
S_{K}(v) & :=\frac{\int_{\mathbb{R}^{2 n}}|v(x)-v(y)|^{2} K(x-y) d x d y}{\left(\int_{\Omega}|v(x)|^{2^{*}} d x\right)^{2 / 2^{*}}} \\
& =\frac{\int_{\mathbb{R}^{2 n}}|v(x)-v(y)|^{2} K(x-y) d x d y}{\left(\int_{\mathbb{R}^{n}}|v(x)|^{2^{*}} d x\right)^{2 / 2^{*}}} .
\end{aligned}
$$

Along the paper we also need the constant

$$
S_{K, \lambda}:=\inf _{v \in X_{0} \backslash\{0\}} S_{K, \lambda}(v),
$$

where for any $v \in X_{0} \backslash\{0\}$

$$
\begin{aligned}
S_{K, \lambda}(v) & :=\frac{\int_{\mathbb{R}^{2 n}}|v(x)-v(y)|^{2} K(x-y) d x d y-\lambda \int_{\Omega}|v(x)|^{2} d x}{\left(\int_{\Omega}|v(x)|^{2^{*}} d x\right)^{2 / 2^{*}}} \\
= & \frac{\int_{\mathbb{R}^{2 n}}|v(x)-v(y)|^{2} K(x-y) d x d y-\lambda \int_{\mathbb{R}^{n}}|v(x)|^{2} d x}{\left(\int_{\mathbb{R}^{n}}|v(x)|^{2^{*}} d x\right)^{2 / 2^{*}}} .
\end{aligned}
$$

Of course, $S_{K, \lambda}(v)<S_{K}(v)$ for any $v \in X_{0} \backslash\{0\}$ and any $\lambda>0$, so $S_{K, \lambda} \leqslant S_{K}$. The topic of research of this paper will actually focus on the case when the strict inequality occurs (see (1.27) below).

In the usual fractional Sobolev space $H^{s}\left(\mathbb{R}^{n}\right)$ the counterpart of $S_{K}$ and $S_{K, \lambda}$ are the constants $S_{s}$ and $S_{s, \lambda}$ defined as follows:

$$
S_{s}:=\inf _{v \in H^{s}\left(\mathbb{R}^{n}\right) \backslash\{0\}} S_{s}(v),
$$


where for any $v \in H^{s}\left(\mathbb{R}^{n}\right) \backslash\{0\}$

$$
S_{s}(v):=\frac{\int_{\mathbb{R}^{2 n}} \frac{|v(x)-v(y)|^{2}}{|x-y|^{n+2 s}} d x d y}{\left(\int_{\mathbb{R}^{n}}|v(x)|^{2^{*}} d x\right)^{2 / 2^{*}}},
$$

and

$$
S_{s, \lambda}:=\inf _{v \in H^{s}\left(\mathbb{R}^{n}\right) \backslash\{0\}} S_{s, \lambda}(v),
$$

where for any $v \in H^{s}\left(\mathbb{R}^{n}\right) \backslash\{0\}$

$$
S_{s, \lambda}(v):=\frac{\int_{\mathbb{R}^{2 n}} \frac{|v(x)-v(y)|^{2}}{|x-y|^{n+2 s}} d x d y-\lambda \int_{\mathbb{R}^{n}}|v(x)|^{2} d x}{\left(\int_{\mathbb{R}^{n}}|v(x)|^{2^{*}} d x\right)^{2 / 2^{*}}} .
$$

We stress that $S_{K, \lambda}$ and $S_{K}$ depend on $\Omega$ (since so does $X_{0}$ ), while $S_{s, \lambda}$ and $S_{s}$ do not (because the minimization occurs on the whole of $H^{s}\left(\mathbb{R}^{n}\right)$ ). The consistency of all these definitions will be discussed in later results (see Lemmas 9, 10, 13 and 14 in Subsection 2.3). Notice that $S_{s, \lambda}(v)<S_{s}(v)$ for any $v \in H^{s}\left(\mathbb{R}^{n}\right) \backslash\{0\}$ and any $\lambda>0$, so $S_{s, \lambda} \leqslant S_{s}$. In the forthcoming Theorems 3 and 4 we will consider the case in which the strict inequality occurs.

The first result of the present paper is the following one:

Theorem 1. Let $s \in(0,1), n>2 s$ and $\Omega$ be an open bounded set of $\mathbb{R}^{n}$ with Lipschitz boundary and let $\lambda \in\left(0, \lambda_{1}\right)$, where $\lambda_{1}$ is the first eigenvalue of the nonlocal operator $-\mathcal{L}_{K}$ with homogeneous Dirichlet boundary conditions.

Let $K: \mathbb{R}^{n} \backslash\{0\} \rightarrow(0,+\infty)$ be a function satisfying conditions (1.7)-(1.9) and let $f: \Omega \times \mathbb{R} \rightarrow \mathbb{R}$ be a Carathéodory function verifying (1.10)-(1.12). Finally, assume that

$$
\text { there exists } u_{0} \in X_{0} \backslash\{0\} \text { with } u_{0} \geqslant 0 \text { a.e. in } \mathbb{R}^{n} \text {, such that }
$$

$$
\sup _{\zeta \geqslant 0} \mathcal{J}_{K, \lambda}\left(\zeta u_{0}\right)<\frac{s}{n} S_{K}^{n /(2 s)}
$$

Then, problem (1.13) admits a solution $u \in X_{0}$, which is not identically zero.

In the case when there is no perturbation of the critical power non-linearity, that is, $f \equiv 0$ in $\Omega \times \mathbb{R}$, problem (1.13) becomes the critical equation

$$
\left\{\begin{array}{c}
\int_{\mathbb{R}^{2 n}}(u(x)-u(y))(\varphi(x)-\varphi(y)) K(x-y) d x d y-\lambda \int_{\Omega} u(x) \varphi(x) d x \\
=\int_{\Omega}|u(x)|^{2^{*}-2} u(x) \varphi(x) d x \quad \forall \varphi \in X_{0} \\
u \in X_{0},
\end{array}\right.
$$

for which Theorem 1 yields the following existence result:

Theorem 2. Let $s \in(0,1), n>2 s$ and $\Omega$ be an open bounded set of $\mathbb{R}^{n}$ with Lipschitz boundary. Let $K: \mathbb{R}^{n} \backslash\{0\} \rightarrow(0,+\infty)$ be a function satisfying conditions (1.7)-(1.9), and let $\lambda \in\left(0, \lambda_{1}\right)$, where $\lambda_{1}$ denotes the first eigenvalue of the 
non-local operator $-\mathcal{L}_{K}$ with homogeneous Dirichlet boundary conditions. Assume that

$$
S_{K, \lambda}<S_{K}
$$

Then, problem (1.26) admits a solution $u \in X_{0}$, which is not identically zero.

In a sense, Theorem 2 clarifies condition (1.25): indeed, as we will see in Section 3.2. condition (1.25), which looks artificial at a first glance, boils down to the more natural condition (1.27) when $f \equiv 0$.

In the non-local framework, the simplest example we can deal with is given by the fractional Laplacian $(-\Delta)^{s}$, for which we have the following critical equation with a lower order perturbation:

$$
\left\{\begin{array}{c}
\int_{\mathbb{R}^{2 n}} \frac{(u(x)-u(y))(\varphi(x)-\varphi(y))}{|x-y|^{n+2 s}} d x d y-\lambda \int_{\Omega} u(x) \varphi(x) d x \\
=\int_{\Omega}|u(x)|^{2^{*}-2} u(x) \varphi(x) d x+\int_{\Omega} f(x, u(x)) \varphi(x) d x \\
\forall \varphi \in H^{s}\left(\mathbb{R}^{n}\right) \text { with } \varphi=0 \text { a.e. in } \mathbb{R}^{n} \backslash \Omega, \\
u \in H^{s}\left(\mathbb{R}^{n}\right) .
\end{array}\right.
$$

In this setting our existence result reads as follows:

Theorem 3. Let $s \in(0,1), n>2 s$ and $\Omega$ be an open bounded set of $\mathbb{R}^{n}$ with Lipschitz boundary, and let $\lambda \in\left(0, \lambda_{1, s}\right)$, where $\lambda_{1, s}$ is the first eigenvalue of the non-local operator $(-\Delta)^{s}$ with homogeneous Dirichlet boundary datum. Let $f$ : $\Omega \times \mathbb{R} \rightarrow \mathbb{R}$ be a Carathéodory function verifying (1.10)-(1.12) and assume that

$$
\begin{gathered}
\text { there exists } u_{0} \in H^{s}\left(\mathbb{R}^{n}\right) \backslash\{0\} \text { with } u_{0} \geqslant 0 \text { a.e. in } \Omega \\
\text { and } u_{0}=0 \text { a.e. in } \mathbb{R}^{n} \backslash \Omega \text {, such that } \\
\sup _{\zeta \geqslant 0} \mathcal{J}_{s, \lambda}\left(\zeta u_{0}\right)<\frac{s}{n} S_{s}^{n /(2 s)} .
\end{gathered}
$$

Then, problem (1.28) admits a solution $u \in H^{s}\left(\mathbb{R}^{n}\right)$, which is not identically zero, such that $u=0$ a.e. in $\mathbb{R}^{n} \backslash \Omega$.

When $f \equiv 0$ in $\Omega \times \mathbb{R}$ problem (1.28) becomes our critical model equation (1.2), whose weak formulation is given by

$$
\left\{\begin{array}{c}
\int_{\mathbb{R}^{2 n}} \frac{(u(x)-u(y))(\varphi(x)-\varphi(y))}{|x-y|^{n+2 s}} d x d y-\lambda \int_{\Omega} u(x) \varphi(x) d x \\
=\int_{\Omega}|u(x)|^{2^{*}-2} u(x) \varphi(x) d x \\
\forall \varphi \in H^{s}\left(\mathbb{R}^{n}\right) \text { with } \varphi=0 \text { a.e. in } \mathbb{R}^{n} \backslash \Omega, \\
u \in H^{s}\left(\mathbb{R}^{n}\right) .
\end{array}\right.
$$


In this framework, assumption (1.29) may be checked directly and Theorem 3 gives:

Theorem 4. Let $s \in(0,1), n \geqslant 4 s$ and $\Omega$ be an open bounded set of $\mathbb{R}^{n}$ with Lipschitz boundary.

Then, denoting by $\lambda_{1, s}$ the first eigenvalue of the non-local operator $(-\Delta)^{s}$ with homogeneous Dirichlet boundary datum, for any $\lambda \in\left(0, \lambda_{1, s}\right)$ problem (1.30) admits a solution $u \in H^{s}\left(\mathbb{R}^{n}\right)$, which is not identically zero, such that $u=0$ a.e. in $\mathbb{R}^{n} \backslash \Omega$.

For the proof of Theorem 4 we use the fact the infimum in (1.21) is attained by a suitable function $\widetilde{u}$, as it is proved in [6, Theorem 1.1]. Then, starting from $\widetilde{u}$ we define a family $u_{\varepsilon}$ and we estimate $S_{s, \lambda}\left(u_{\varepsilon}\right)$; see Subsection 4.2 for the details. With respect to the classical case of the Laplacian, here this estimate is more delicate, due to the non-local nature of the operator $(-\Delta)^{s}$. In particular, in the non-local framework, in order to control the Gagliardo seminorm of $u_{\varepsilon}$, pointwise estimates on $u_{\varepsilon}$ are not enough. Indeed, we also need some integral estimates which allow us to control the interactions between $B_{\delta}$ and $\mathbb{R}^{n} \backslash B_{\delta}$ (for more details, see Proposition 21).

As we said before, in this paper we adapt the variational approach used in 3 . to the non-local framework. For this we will work in a functional analytical setting that is inspired by (but not equivalent to) the fractional Sobolev spaces, in order to correctly encode the Dirichlet boundary datum in the variational formulation. Also the functional setting we will use allows us to overcome the problems related to the lack of compactness and to show that the Palais-Smale condition holds true in a suitable range related to the best fractional Sobolev constant in the embedding $X_{0} \hookrightarrow L^{2^{*}}\left(\mathbb{R}^{n}\right)$ (or, in the case of the fractional Laplacian, $H^{s}\left(\mathbb{R}^{n}\right) \hookrightarrow L^{2^{*}}\left(\mathbb{R}^{n}\right)$ ); see Subsection 4.2 .2 for more details. In fact, this range of validity of the Palais-Smale condition may be explicitly related to the strict sign of the inequality obtained from the Sobolev constants, as we discuss in Proposition 23.

Finally, note that when $s=1$ Theorem 4 reads as part $a$ ) of the results obtained in [3] for the Laplacian. For all these reasons we think that Theorem 4 may be seen as the natural extension of the results proved in [3] (see also [16, 18]) to the non-local fractional framework. With this respect, we recall the recent paper [17, where a non-local version of the Brezis-Nirenberg result was also considered (in the case $s=1 / 2$ and for an operator conceptually different than the one treated here). For results similar to the ones presented here but in low dimension, we refer to [12].

The paper is organized as follows. In Section 2 we give some basic estimates on the non-linearity $f$ and its primitive. Moreover, we introduce the functional setting we will work in and we discuss some embeddings of the spaces $X_{0}$ and $H^{s}\left(\mathbb{R}^{n}\right)$ into the usual Lebesgue spaces. At the end of the section, we deal with an eigenvalue problem driven by the non-local integrodifferential operator $-\mathcal{L}_{K}$ and we recall some properties of its first eigenvalue. In Section 3 we prove Theorems 11 and 2 by performing a variational method (in fact, we reduce Theorem 2 to Theorem 1 proving the equivalence of conditions (1.25) and (1.27) when $f \equiv 0)$. Finally, in Section 4, as an application, we consider the case of an equation driven by the fractional Laplacian operator and we prove Theorems 3 and 4 (for this, condition (1.29) will be reduced to (1.25), and so Theorem 1 will be applied to prove Theorem 3 , which, in turn, will imply Theorem 4).

The proofs are, in several points, different than the ones of the classical case of the Laplacian. These additional complications are not only technical, but, sometimes, they somehow reflect the different distribution of the energy density due to the 
non-local interactions. For instance, the far-away part of the energy in our case, though it is small, gives a contribution to the functional that cannot be neglected, since it is of order comparable with the perturbation.

\section{Some PRELIMINARY RESUlts}

In this section we prove some preliminary results which will be useful in the sequel.

2.1. Estimates on the non-linearity and its primitive. Here we gather some elementary results which will be useful in the main estimates of the paper. We use the behavior of $f$ at zero and at infinity in order to deduce some estimates on the non-linear term and its primitive (with respect to its second variable) $F$ defined in (1.16). This part is quite standard and does not take into account the non-local nature of the problem; the reader familiar with these topics may skip it and go directly to Subsection 2.2 ,

Lemma 5. Assume $f: \Omega \times \mathbb{R} \rightarrow \mathbb{R}$ is a Carathéodory function satisfying conditions (1.10)-(1.12). Then, for any $\varepsilon>0$ there exists $M=M(\varepsilon)>0$ such that a.e. $x \in \Omega$ and for any $t \in \mathbb{R}$

$$
|f(x, t)| \leqslant 2^{*} \varepsilon|t|^{2^{*}-1}+M(\varepsilon),
$$

and so, as a consequence,

$$
|F(x, t)| \leqslant \varepsilon|t|^{2^{*}}+M(\varepsilon)|t|,
$$

where $F$ is defined as in (1.16).

Proof. By assumption (1.12) for any $\varepsilon>0$ there exists $\sigma=\sigma(\varepsilon)>0$ such that for any $t \in \mathbb{R}$ with $|t|>\sigma$ and a.e. $x \in \Omega$ we get

$$
|f(x, t)| \leqslant 2^{*} \varepsilon|t|^{2^{*}-1} .
$$

Moreover, by (1.10) there exists $M(\varepsilon)=M(\sigma(\varepsilon))>0$ such that a.e. $x \in \Omega$ and for any $t \in \mathbb{R}$ with $|t| \leqslant \sigma$ we have

$$
|f(x, t)| \leqslant M(\varepsilon) .
$$

Combining (2.3) and (2.4) it easily follows (2.1). Using the definition of $F$ (see (1.16), we also get (2.2)

Lemma 6. Assume $f: \Omega \times \mathbb{R} \rightarrow \mathbb{R}$ is a Carathéodory function satisfying conditions (1.10) -(1.12). Then, for any $\varepsilon>0$ there exists $\delta=\delta(\varepsilon)$ such that a.e. $x \in \Omega$ and for any $t \in \mathbb{R}$

$$
|f(x, t)| \leqslant 2 \varepsilon|t|+2^{*} \delta(\varepsilon)|t|^{2^{*}-1},
$$

and so, as a consequence,

$$
|F(x, t)| \leqslant \varepsilon|t|^{2}+\delta(\varepsilon)|t|^{2^{*}},
$$

where $F$ is defined as in (1.16).

Proof. By assumption (1.11) for any $\varepsilon>0$ there exists $\sigma=\sigma(\varepsilon)>0$ such that for any $t \in \mathbb{R}$ with $|t|<\sigma$ and a.e. $x \in \Omega$ we get

$$
|f(x, t)| \leqslant 2 \varepsilon|t| \text {. }
$$


Moreover, by (2.1) with $\varepsilon=1$ we get that

$$
|f(x, t)| \leqslant 2^{*}|t|^{2^{*}-1}+M(1)=|t|^{2^{*}-1}\left(2^{*}+\frac{M(1)}{|t|^{2^{*}-1}}\right),
$$

so that there exists $\delta(\varepsilon)=\delta(\sigma(\varepsilon))>0$ such that a.e. $x \in \Omega$ and for any $t \in \mathbb{R}$ with $|t| \geqslant \sigma$ we have

$$
|f(x, t)| \leqslant 2^{*} \delta(\varepsilon)|t|^{2^{*}-1} .
$$

Combining (2.7) and (2.8) it easily follows (2.5). Using the definition of $F$ given in (1.16), we also deduce (2.6).

2.2. The functional setting. Here, we give some basic results on the spaces $X$ and $X_{0}$. The reader familiar with the fractional Sobolev spaces and their extensions (as in [14, 15]) may go directly to Section [3. In the sequel we set $Q=\mathbb{R}^{2 n} \backslash \mathcal{O}$, where

and $\mathcal{C} \Omega=\mathbb{R}^{n} \backslash \Omega$.

$$
\mathcal{O}=(\mathcal{C} \Omega) \times(\mathcal{C} \Omega) \subset \mathbb{R}^{2 n}
$$

The space $X$ is endowed with the norm defined as

$$
\|g\|_{X}=\|g\|_{L^{2}(\Omega)}+\left(\int_{Q}|g(x)-g(y)|^{2} K(x-y) d x d y\right)^{1 / 2} .
$$

It is easily seen that $\|\cdot\|_{X}$ is a norm on $X$ (see, for instance, 14 for a proof).

In the following we denote by $H^{s}(\Omega)$ the usual fractional Sobolev space endowed with the norm (the so-called Gagliardo norm)

$$
\|g\|_{H^{s}(\Omega)}=\|g\|_{L^{2}(\Omega)}+\left(\int_{\Omega \times \Omega} \frac{|g(x)-g(y)|^{2}}{|x-y|^{n+2 s}} d x d y\right)^{1 / 2} .
$$

We remark that, even in the model case in which $K(x)=|x|^{-(n+2 s)}$, the norms in (2.9) and (2.10) are not the same, because $\Omega \times \Omega$ is strictly contained in $Q$ (this makes the classical fractional Sobolev space approach not sufficient for studying the problem).

For further details on the fractional Sobolev spaces we refer to 9 and to the references therein.

In the next result we give some connections between the space $X_{0}$ and the usual fractional Sobolev spaces $H^{s}\left(\mathbb{R}^{n}\right)$, which will be useful along the paper.

Lemma 7. The following assertions hold true:

a) Let $K: \mathbb{R}^{n} \backslash\{0\} \rightarrow(0,+\infty)$ satisfy assumptions (1.7)-1.9). Then $X_{0} \subseteq$ $H^{s}\left(\mathbb{R}^{n}\right)$ and, moreover,

$$
\|v\|_{H^{s}(\Omega)} \leqslant\|v\|_{H^{s}\left(\mathbb{R}^{n}\right)} \leqslant c(\theta)\|v\|_{X}
$$

where $c(\theta)=\max \left\{1, \theta^{-1 / 2}\right\}$, with $\theta$ given in (1.8).

b) Let $K(x)=|x|^{-(n+2 s)}$. Then

$$
X_{0}=\left\{v \in H^{s}\left(\mathbb{R}^{n}\right): v=0 \text { a.e. in } \mathbb{R}^{n} \backslash \Omega\right\} .
$$

Proof. Part $a$ ) was proved in [14, Lemma 5-b)]. Let us show part $b$ ). By assertion $a$ ) it is easily seen that $X_{0} \subseteq\left\{v \in H^{s}\left(\mathbb{R}^{n}\right): v=0\right.$ a.e. in $\left.\mathbb{R}^{n} \backslash \Omega\right\}$. 
Conversely, let $v \in H^{s}\left(\mathbb{R}^{n}\right)$ be such that $v=0$ a.e. in $\mathbb{R}^{n} \backslash \Omega$. Since $v \in H^{s}\left(\mathbb{R}^{n}\right)$, we have that

$$
\int_{\mathbb{R}^{n}}|v(x)|^{2} d x<+\infty
$$

and

$$
\int_{\mathbb{R}^{2 n}} \frac{|v(x)-v(y)|^{2}}{|x-y|^{n+2 s}} d x d y<+\infty,
$$

from which, using the property that $v=0$ a.e. in $\mathbb{R}^{n} \backslash \Omega$, we deduce

$$
\int_{\Omega}|v(x)|^{2} d x=\int_{\mathbb{R}^{n}}|v(x)|^{2} d x<+\infty
$$

and

$$
\int_{Q} \frac{|v(x)-v(y)|^{2}}{|x-y|^{n+2 s}} d x d y=\int_{\mathbb{R}^{2 n}} \frac{|v(x)-v(y)|^{2}}{|x-y|^{n+2 s}} d x d y<+\infty .
$$

Hence, $v \in X_{0}$, and this ends the proof of Lemma 7 b).

In the sequel we consider the function

$$
X_{0} \ni v \mapsto\|v\|_{X_{0}}=\left(\int_{Q}|v(x)-v(y)|^{2} K(x-y) d x d y\right)^{1 / 2}
$$

and we take (2.11) as a norm on $X_{0}$. The following result, proved in [14, Lemmas 6 and 7], holds true:

Lemma 8. Let $K: \mathbb{R}^{n} \backslash\{0\} \rightarrow(0,+\infty)$ satisfy assumptions (1.7)-(1.9). Then

a) there exists a positive constant $c$, depending only on $n$ and $s$, such that for any $v \in X_{0}$

$$
\|v\|_{L^{2^{*}}(\Omega)}^{2}=\|v\|_{L^{2^{*}\left(\mathbb{R}^{n}\right)}}^{2} \leqslant c \int_{\mathbb{R}^{2 n}} \frac{|v(x)-v(y)|^{2}}{|x-y|^{n+2 s}} d x d y,
$$

where $2^{*}$ is given in (1.4);

b) there exists a constant $C>1$, depending only on $n, s, \theta$ and $\Omega$, such that for any $v \in X_{0}$

$$
\int_{Q}|v(x)-v(y)|^{2} K(x-y) d x d y \leqslant\|v\|_{X}^{2} \leqslant C \int_{Q}|v(x)-v(y)|^{2} K(x-y) d x d y,
$$

that is, formula (2.11) defines a norm on $X_{0}$ equivalent to the usual one given in (2.9);

c) $\left(X_{0},\|\cdot\|_{X_{0}}\right)$ is a Hilbert space, with scalar product

$$
\langle u, v\rangle_{X_{0}}=\int_{Q}(u(x)-u(y))(v(x)-v(y)) K(x-y) d x d y .
$$

Note that in (2.11) (and in the related scalar product; see Lemma 8tc)) the integral can be extended to all $\mathbb{R}^{2 n}$, since $v \in X_{0}$ (and so $v=0$ a.e. in $\mathbb{R}^{n} \backslash \Omega$ ). 
2.3. Some embeddings into the usual Lebesgue spaces. In this subsection we discuss some results related to the embeddings of the spaces $X_{0}$ and $H^{s}\left(\mathbb{R}^{n}\right)$ into the usual Lebesgue spaces, which allow us to define the best fractional critical Sobolev constants given in formulas (1.17), (1.19), (1.21) and (1.23).

Lemma 9. Let $K: \mathbb{R}^{n} \backslash\{0\} \rightarrow(0,+\infty)$ satisfy assumptions (1.7)-(1.9). Then, the following assertions hold true:

a) if $\Omega$ has a Lipschitz boundary 4 then the embedding $X_{0} \hookrightarrow L^{\nu}\left(\mathbb{R}^{n}\right)$ is compact for any $\nu \in\left[1,2^{*}\right)$;

b) the embedding $X_{0} \hookrightarrow L^{2^{*}}\left(\mathbb{R}^{n}\right)$ is continuous.

Proof. Part $a$ ) is proved, for instance, in [14, Lemma 8]. Let us show $b$ ). For this, by Lemma 8 - $a$ ) and assumption (1.8) we get that for any $v \in X_{0}$

$$
\begin{aligned}
\|v\|_{L^{2^{*}}(\Omega)}^{2}=\|v\|_{L^{2^{*}\left(\mathbb{R}^{n}\right)}}^{2} & \leqslant c \int_{\mathbb{R}^{2 n}} \frac{|v(x)-v(y)|^{2}}{|x-y|^{n+2 s}} d x d y \\
& \leqslant \frac{c}{\theta} \int_{\mathbb{R}^{2 n}}|v(x)-v(y)|^{2} K(x-y) d x d y \\
& =\frac{c}{\theta}\|v\|_{X_{0}}^{2},
\end{aligned}
$$

for a suitable positive constant $c$, depending only on $n$ and $s$. Hence assertion $b$ ) is proved.

Thanks to Lemma 9 we can define the constant $S_{K}$ given in formula (1.17) and see that $S_{K}>0$. Moreover, note that, since in formula (1.18) the integral over $\Omega$ can be extended to all $\mathbb{R}^{n}$ (being $v=0$ a.e. in $\mathbb{R}^{n} \backslash \Omega$ ), then the function $v \mapsto S_{K}(v)$ does not depend on the domain $\Omega$, while, in general, $S_{K}$ does (indeed, $X_{0}$ depends on $\Omega$ ).

The counterpart of Lemma 9 in the usual fractional Sobolev spaces is given by the following result proved, for example, in [9, Theorem 6.5]:

Lemma 10. The embedding $H^{s}\left(\mathbb{R}^{n}\right) \hookrightarrow L^{\nu}\left(\mathbb{R}^{n}\right)$ is continuous for any $\nu \in\left[2,2^{*}\right]$.

Lemma 10 allows us to define the constant $S_{s}$ given in formula (1.21) and see that $S_{s}>0$.

Remark 11. In the particular case when $K(x)=|x|^{-(n+2 s)}$, it is easily seen that

$$
\inf _{v \in H^{s}\left(\mathbb{R}^{n}\right) \backslash\{0\}} S_{s}(v) \leqslant \inf _{v \in X_{0} \backslash\{0\}} S_{K}(v),
$$

since $X_{0} \subseteq H^{s}\left(\mathbb{R}^{n}\right)$ by Lemma $\left.7 \rightarrow a\right)$.

As a final observation, we note that, with respect to the embeddings in the Lebesgue spaces, the fractional Sobolev space $H^{s}\left(\mathbb{R}^{n}\right)$ behaves like the usual Sobolev space $H^{1}\left(\mathbb{R}^{n}\right)$, while $X_{0}$ like $H_{0}^{1}(\Omega)$ (this is due to the fact that the functions $v \in X_{0}$ are such that $v=0$ a.e. in $\mathbb{R}^{n} \backslash \Omega$, and so $X_{0}$ may be seen somehow as a space of functions defined in the bounded set $\Omega$ ).

\footnotetext{
${ }^{4}$ Here the regularity of the domain $\Omega$ can be weakened. Indeed, thanks to [7, Theorem 6$]$, it is enough to assume that $\Omega$ is a bounded subset of $\mathbb{R}^{n}$ with continuous boundary.
} 
Due to the presence of the term $\lambda u$ in the equations we are interested in, it is convenient to define another norm in the spaces $X_{0}$ and $H^{s}\left(\mathbb{R}^{n}\right)$, respectively, as follows:

$$
X_{0} \ni v \mapsto\|v\|_{X_{0}, \lambda}:=\left(\int_{\mathbb{R}^{2 n}}|v(x)-v(y)|^{2} K(x-y) d x d y-\lambda \int_{\Omega}|v(x)|^{2} d x\right)^{1 / 2},
$$

and

$$
H^{s}\left(\mathbb{R}^{n}\right) \ni v \mapsto\|v\|_{H^{s}\left(\mathbb{R}^{n}\right), \lambda}:=\left(\int_{\mathbb{R}^{2 n}} \frac{|v(x)-v(y)|^{2}}{|x-y|^{n+2 s}} d x d y-\lambda \int_{\mathbb{R}^{n}}|v(x)|^{2} d x\right)^{1 / 2} .
$$

In [15. Lemma 10] we proved the following result:

Lemma 12. Let $K: \mathbb{R}^{n} \backslash\{0\} \rightarrow(0,+\infty)$ satisfy assumptions (1.7)-(1.9) and let $\lambda \in\left(0, \lambda_{1}\right)$, where $\lambda_{1}$ is the first eigenvalue of the operator $-\mathcal{L}_{K}$ with homogeneous Dirichlet boundary conditions. Then, formula (2.12) defines a norm on $X_{0}$ which is equivalent to the usual one given in (2.11) in the sense that for any $v \in X_{0}$

$$
\left(1-\frac{\lambda}{\lambda_{1}}\right)\|v\|_{X_{0}}^{2} \leqslant\|v\|_{X_{0}, \lambda}^{2} \leqslant\|v\|_{X_{0}}^{2} .
$$

As a consequence of Lemmas $9+b)$ and 12 we have the following result:

Lemma 13. Let $K: \mathbb{R}^{n} \backslash\{0\} \rightarrow(0,+\infty)$ satisfy assumptions (1.7)-(1.9) and let $\lambda \in\left(0, \lambda_{1}\right)$, where $\lambda_{1}$ is the first eigenvalue of the operator $-\mathcal{L}_{K}$ with homogeneous Dirichlet boundary conditions. Then the embedding $\left(X_{0},\|\cdot\|_{X_{0}, \lambda}\right) \hookrightarrow L^{2^{*}}\left(\mathbb{R}^{n}\right)$ is continuous.

Thanks to Lemma 13 we can define the constant $S_{K, \lambda}$ as in formula (1.19) and get that $S_{K, \lambda}>0$. Also in this case we note that, since in formula (1.20) the integral over $\Omega$ can be extended to all $\mathbb{R}^{n}$ (being $v=0$ a.e. in $\mathbb{R}^{n} \backslash \Omega$ ), then the function $v \mapsto S_{K, \lambda}(v)$ does not depend on the domain $\Omega$, while, in general, $S_{K, \lambda}$ does.

The counterpart of Lemmas 12 and 13 in the usual fractional Sobolev spaces is given by the following result:

Lemma 14. Let $\lambda \in\left(0, \lambda_{1, s}\right)$, where $\lambda_{1, s}$ is the first eigenvalue of the operator $(-\Delta)^{s}$ with homogeneous Dirichlet boundary conditions. Then formula (2.13) defines a norm on $H^{s}\left(\mathbb{R}^{n}\right)$ equivalent to the usual one given in (2.10).

Moreover, the embedding $\left(H^{s}\left(\mathbb{R}^{n}\right),\|\cdot\|_{H^{s}\left(\mathbb{R}^{n}\right), \lambda}\right) \hookrightarrow L^{2^{*}}\left(\mathbb{R}^{n}\right)$ is continuous.

Proof. To see that formula (2.13) defines a norm on $H^{s}\left(\mathbb{R}^{n}\right)$ equivalent to the usual one, it is enough to argue as in the proof of [15, Lemma 10].

The continuity of the embedding $\left(H^{s}\left(\mathbb{R}^{n}\right),\|\cdot\|_{H^{s}\left(\mathbb{R}^{n}\right), \lambda}\right) \hookrightarrow L^{2^{*}}\left(\mathbb{R}^{n}\right)$ comes from the fact that the norms (2.10) and (2.13) are equivalent and by Lemma 10.

Thanks to Lemma 14 we can define the constant $S_{s, \lambda}$ given in formula (1.23), and conclude that $S_{s, \lambda}>0$. 
Remark 15. In the model case when $K(x)=|x|^{-(n+2 s)}$, it is easy to prove that

$$
\inf _{v \in H^{s}\left(\mathbb{R}^{n}\right) \backslash\{0\}} S_{s, \lambda}(v) \leqslant \inf _{v \in X_{0} \backslash\{0\}} S_{K, \lambda}(v),
$$

since $X_{0} \subseteq H^{s}\left(\mathbb{R}^{n}\right)$ by Lemma 7 f $\left.a\right)$.

Remark 16. In the case when $\lambda=0$, we get that

$$
S_{K, \lambda}=S_{K} \quad \text { and } \quad S_{s, \lambda}=S_{s},
$$

while for $\lambda>0$ we have that

$$
S_{K, \lambda} \leqslant S_{K} \quad \text { and } \quad S_{s, \lambda} \leqslant S_{s} .
$$

2.4. An eigenvalue problem. Here we consider the following eigenvalue problem driven by the non-local integrodifferential operator $-\mathcal{L}_{K}$ :

$$
\begin{cases}-\mathcal{L}_{K} u=\lambda u & \text { in } \Omega, \\ u=0 & \text { in } \mathbb{R}^{n} \backslash \Omega,\end{cases}
$$

where $s \in(0,1), n>2 s, \Omega$ is an open bounded set of $\mathbb{R}^{n}$ and $K: \mathbb{R}^{n} \backslash\{0\} \rightarrow(0,+\infty)$ is a function satisfying (1.7)- (1.9).

More precisely, we are interested in the weak formulation of (2.15), which consists of the following eigenvalue problem:

$$
\left\{\begin{array}{c}
\int_{\mathbb{R}^{2 n}}(u(x)-u(y))(\varphi(x)-\varphi(y)) K(x-y) d x d y \\
=\lambda \int_{\Omega} u(x) \varphi(x) d x \quad \forall \varphi \in X_{0}, \\
u \in X_{0} .
\end{array}\right.
$$

We recall that $\lambda \in \mathbb{R}$ is an eigenvalue of $-\mathcal{L}_{K}$ provided there exists a nontrivial solution $u \in X_{0}$ of problem (2.15) - in fact, of its weak formulation (2.16) and, in this case, any solution will be called an eigenfunction corresponding to the eigenvalue $\lambda$.

For the purpose of this paper we need only the properties of the first eigenvalue of $-\mathcal{L}_{K}$ and of its eigenfunction, that is, the following result, whose proof can be found in [15, Proposition 9 and Appendix A], where a complete study of the spectrum of $-\mathcal{L}_{K}$ is presented:

Proposition 17 (First eigenvalue and first eigenfunction of $-\mathcal{L}_{K}$ ). Let $s \in(0,1)$, $n>2 s, \Omega$ be an open bounded set of $\mathbb{R}^{n}$ and let $K: \mathbb{R}^{n} \backslash\{0\} \rightarrow(0,+\infty)$ be a function satisfying assumptions (1.7)-(1.9). Then,

a) problem (2.16) admits an eigenvalue $\lambda_{1}$ which is positive and that can be characterized as

$$
\lambda_{1}=\min _{\substack{u \in X_{0} \\\|u\|_{L^{2}(\Omega)}=1}} \int_{\mathbb{R}^{2 n}}|u(x)-u(y)|^{2} K(x-y) d x d y,
$$

or, equivalently,

$$
\lambda_{1}=\min _{u \in X_{0} \backslash\{0\}} \frac{\int_{\mathbb{R}^{2 n}}|u(x)-u(y)|^{2} K(x-y) d x d y}{\int_{\Omega}|u(x)|^{2} d x} ;
$$


b) there exists a non-negative function $e_{1} \in X_{0}$, which is an eigenfunction corresponding to $\lambda_{1}$, attaining the minimum in (2.17), that is, $\left\|e_{1}\right\|_{L^{2}(\Omega)}=$ 1 and

$$
\lambda_{1}=\int_{\mathbb{R}^{2 n}}\left|e_{1}(x)-e_{1}(y)\right|^{2} K(x-y) d x d y
$$

c) $\lambda_{1}$ is simple; that is, if $u \in X_{0}$ is a solution of the equation

$$
\int_{\mathbb{R}^{2 n}}(u(x)-u(y))(\varphi(x)-\varphi(y)) K(x-y) d x d y=\lambda_{1} \int_{\Omega} u(x) \varphi(x) d x \quad \forall \varphi \in X_{0},
$$

then $u=\zeta e_{1}$, with $\zeta \in \mathbb{R}$.

\section{A general CRitical integrodifFerential EQUATion}

In this section we study problem (1.13) which is the Euler-Lagrange equation of the functional $\mathcal{J}_{K, \lambda}$ defined in (1.15). Notice that this functional is well defined thanks to assumptions (1.10) and (1.12), to the fact that $\Omega$ is bounded, and to Lemma 9 $b$ ). Moreover, $\mathcal{J}_{K, \lambda}$ is Fréchet differentiable in $u \in X_{0}$, and for any $\varphi \in X_{0}$

$$
\begin{array}{r}
\left\langle\mathcal{J}_{K, \lambda}^{\prime}(u), \varphi\right\rangle=\int_{\mathbb{R}^{2 n}}(u(x)-u(y))(\varphi(x)-\varphi(y)) K(x-y) d x d y-\lambda \int_{\Omega} u(x) \varphi(x) d x \\
-\int_{\Omega}|u(x)|^{2^{*}-1}(x) \varphi(x) d x-\int_{\Omega} f(x, u(x)) \varphi(x) d x
\end{array}
$$

Thus, critical points of $\mathcal{J}_{K, \lambda}$ are solutions to problem (1.13). In order to find these critical points, we will apply a variant of the Mountain Pass Theorem without the Palais-Smale condition, as given in [1] (see also [3, Theorem 2.2]).

Indeed, here we can not apply the classical Mountain Pass Theorem, since, due to the lack of compactness in the embedding $X_{0} \hookrightarrow L^{2^{*}}\left(\mathbb{R}^{n}\right)$ (see Lemma 9. b)), the functional $\mathcal{J}_{K, \lambda}$ does not verify the Palais-Smale condition globally, but only in an energy range determined by the best fractional critical Sobolev constant $S_{K}$ given in formula (1.17) (see also Lemma 9-b)).

This variant of the Mountain Pass Theorem requires that the functional $\mathcal{J}_{K, \lambda}$ has a suitable geometric structure, as stated, e.g., in conditions (2.9) and (2.10) of [3. Theorem 2.2].

3.1. The critical case with a lower order perturbation. This subsection is devoted to problem (1.13) and to the proof of Theorem 1.

First of all, we prove that the functional $\mathcal{J}_{K, \lambda}$ has the geometric features required by [3, Theorem 2.2].

Proposition 18. Let $\lambda \in\left(0, \lambda_{1}\right)$ and let $f$ be a Carathéodory function satisfying conditions (1.10)-(1.12). Then, there exist $\rho>0$ and $\beta>0$ such that for any $u \in X_{0}$ with $\|u\|_{X_{0}}=\rho$, it results that $\mathcal{J}_{K, \lambda}(u) \geqslant \beta$. 
Proof. Let $u$ be a function in $X_{0}$. By (2.6) we get that for any $\varepsilon>0$

$$
\begin{aligned}
\mathcal{J}_{K, \lambda}(u) \geqslant \frac{1}{2} & \int_{\mathbb{R}^{2 n}}|u(x)-u(y)|^{2} K(x-y) d x d y-\frac{\lambda}{2} \int_{\Omega}|u(x)|^{2} d x \\
& -\frac{1}{2^{*}} \int_{\Omega}|u(x)|^{2^{*}} d x-\varepsilon \int_{\Omega}|u(x)|^{2} d x-\delta(\varepsilon) \int_{\Omega}|u(x)|^{2^{*}} d x \\
\geqslant & \frac{1}{2}\left(1-\frac{\lambda}{\lambda_{1}}\right) \int_{\mathbb{R}^{2 n}}|u(x)-u(y)|^{2} K(x-y) d x d y-\varepsilon\|u\|_{L^{2}(\Omega)}^{2} \\
& -\left(\frac{1}{2^{*}}+\delta(\varepsilon)\right)\|u\|_{L^{2^{*}}(\Omega)}^{2^{*}} \\
\geqslant & \frac{1}{2}\left(1-\frac{\lambda}{\lambda_{1}}\right) \int_{\mathbb{R}^{2 n}}|u(x)-u(y)|^{2} K(x-y) d x d y \\
& \quad-\varepsilon|\Omega|^{\left(2^{*}-2\right) / 2^{*}}\|u\|_{L^{2 *}(\Omega)}^{2}-\left(\frac{1}{2^{*}}+\delta(\varepsilon)\right)\|u\|_{L^{2^{*}}(\Omega)}^{2^{*}}
\end{aligned}
$$

thanks to Lemma 12 (here we need $0<\lambda<\lambda_{1}$ ) and to the fact that $L^{2^{*}}(\Omega) \hookrightarrow$ $L^{2}(\Omega)$ continuously (being $\Omega$ bounded and $2<2^{*}$ ).

Using (1.8) and Lemma $8 . a)-b$ ), we deduce from (3.1) that for any $\varepsilon>0$

$$
\begin{aligned}
\mathcal{J}_{K, \lambda}(u) \geqslant & \frac{1}{2}\left(1-\frac{\lambda}{\lambda_{1}}\right) \int_{\mathbb{R}^{2 n}}|u(x)-u(y)|^{2} K(x-y) d x d y \\
& -\varepsilon c|\Omega|^{\left(2^{*}-2\right) / 2^{*}} \int_{\mathbb{R}^{2 n}} \frac{|u(x)-u(y)|^{2}}{|x-y|^{n+2 s}} d x d y \\
& -\left(\frac{1}{2^{*}}+\delta(\varepsilon)\right) c^{2^{*} / 2}\left(\int_{\mathbb{R}^{2 n}} \frac{|u(x)-u(y)|^{2}}{|x-y|^{n+2 s}} d x d y\right)^{2^{*} / 2} \\
\geqslant & {\left[\frac{1}{2}\left(1-\frac{\lambda}{\lambda_{1}}\right)-\frac{\varepsilon c|\Omega|^{\left(2^{*}-2\right) / 2^{*}}}{\theta}\right] \int_{\mathbb{R}^{2 n}}|u(x)-u(y)|^{2} K(x-y) d x d y } \\
& -\left(\frac{1}{2^{*}}+\delta(\varepsilon)\right)\left(\frac{c}{\theta}\right)^{2^{*} / 2}\left(\int_{\mathbb{R}^{2 n}}|u(x)-u(y)|^{2} K(x-y) d x d y\right)^{2^{*} / 2} \\
= & {\left[\frac{1}{2}\left(1-\frac{\lambda}{\lambda_{1}}\right)-\frac{\varepsilon c|\Omega|^{\left(2^{*}-2\right) / 2^{*}}}{\theta}\right]\|u\|_{X_{0}}^{2}-\left(\frac{1}{2^{*}}+\delta(\varepsilon)\right)\left(\frac{c}{\theta}\right)^{2^{*} / 2}\|u\|_{X_{0}}^{2^{*}} . }
\end{aligned}
$$
that

Choosing $\varepsilon>0$ such that $2 \varepsilon c|\Omega|^{\left(2^{*}-2\right) / 2^{*}}<\theta\left(1-\lambda / \lambda_{1}\right)$, by (3.2) it easily follows

$$
\mathcal{J}_{K, \lambda}(u) \geqslant \alpha\|u\|_{X_{0}}^{2}\left(1-\kappa\|u\|_{X_{0}}^{2^{*}-2}\right)
$$

for suitable positive constants $\alpha$ and $\kappa$.

Now, let $u \in X_{0}$ be such that $\|u\|_{X_{0}}=\rho>0$. Since $2^{*}>2$, we can choose $\rho$ sufficiently small (i.e. $\rho$ such that $1-\kappa \rho^{2^{*}-2}>0$ ), so that

$$
\inf _{\substack{u \in X_{0} \\\|u\|_{X_{0}}=\rho}} \mathcal{J}_{K, \lambda}(u) \geqslant \alpha \rho^{2}\left(1-\kappa \rho^{2^{*}-2}\right)=: \beta>0 .
$$

Hence, Proposition 18 is proved.

Proposition 19. Let $\lambda \in\left(0, \lambda_{1}\right)$ and let $f$ be a Carathéodory function satisfying conditions (1.10)-(1.12). Then, there exists $e \in X_{0}$ such that $e \geqslant 0$ a.e. in $\mathbb{R}^{n}$, $\|e\|_{X_{0}}>\rho$ and $\mathcal{J}_{K, \lambda}(e)<\beta$, where $\rho$ and $\beta$ are given in Proposition 18 . 
In particular, if we assume condition (1.25), we can construct e as follows:

$$
e=\zeta_{0} u_{0},
$$

with $u_{0}$ as in (1.25) and $\zeta_{0}>0$ large enough.

Proof. We fix $u \in X_{0}$ such that $\|u\|_{X_{0}}=1$ and $u \geqslant 0$ a.e. in $\mathbb{R}^{n}$. We remark that this choice is possible thanks to (1.14) (alternatively, one can replace any $u \in X_{0}$ with its positive part, which belongs to $X_{0}$ too, thanks to [13, Lemma 5.2]).

Now, let $\zeta>0$. By Lemma 12 (here we again use the fact that $\lambda<\lambda_{1}$ ) and Lemma 5 (see formula (2.2) used here with $\varepsilon=1 /\left(2 \cdot 2^{*}\right)$ ) we have

$$
\begin{aligned}
\mathcal{J}_{K, \lambda}(\zeta u)= & \frac{\zeta^{2}}{2} \int_{\mathbb{R}^{2 n}}|u(x)-u(y)|^{2} K(x-y) d x d y-\frac{\lambda}{2} \zeta^{2} \int_{\Omega}|u(x)|^{2} d x \\
& \quad-\frac{\zeta^{2^{*}}}{2^{*}} \int_{\Omega}|u(x)|^{2^{*}} d x-\int_{\Omega} F(x, \zeta u(x)) d x \\
\leqslant & \frac{\zeta^{2}}{2}-\left(\frac{1}{2^{*}}-\frac{1}{2 \cdot 2^{*}}\right) \zeta^{2^{*}} \int_{\Omega}|u(x)|^{2^{*}} d x+M\left(1 /\left(2 \cdot 2^{*}\right)\right) \int_{\Omega}|\zeta u(x)| d x \\
= & \frac{\zeta^{2}}{2}-\frac{\zeta^{2^{*}}}{2 \cdot 2^{*}} \int_{\Omega}|u(x)|^{2^{*}} d x+\zeta M\left(1 /\left(2 \cdot 2^{*}\right)\right) \int_{\Omega}|u(x)| d x .
\end{aligned}
$$

Since $2^{*}>2>1$, passing to the limit as $\zeta \rightarrow+\infty$, we get that $\mathcal{J}_{K, \lambda}(\zeta u) \rightarrow-\infty$, so that the assertion follows taking $e=\zeta u$, with $\zeta$ sufficiently large.

In particular, under condition (1.25), we can take $u=u_{0} /\left\|u_{0}\right\|_{X_{0}} \in X_{0}$ (note that $u_{0} \not \equiv 0$ by assumption) so that we can choose $e=\zeta_{0} u_{0}$ with $\zeta_{0}$ large enough.

3.1.1. End of the proof of Theorem 1. Propositions 18 and 19 give that the geometry of the variant of the Mountain Pass Theorem stated in [3, Theorem 2.2] is fulfilled by $\mathcal{J}_{K, \lambda}$. Moreover, since $F(0)=0$, we easily get that $\mathcal{J}_{K, \lambda}(0)=0<\beta$, with $\beta$ given in Proposition 18 . Now, set

$$
c=\inf _{P \in \mathcal{P}} \sup _{v \in P([0,1])} \mathcal{J}_{K, \lambda}(v),
$$

where

$$
\mathcal{P}=\left\{P \in C\left([0,1] ; X_{0}\right): P(0)=0, P(1)=e\right\}
$$

with $e=\zeta_{0} u_{0}$ given in Proposition [19] In order to prove Theorem 1 we proceed by steps.

Claim 1. The constant $c$ given in (3.4) is such that

$$
\beta \leqslant c<\frac{s}{n} S_{K}^{n /(2 s)},
$$

where $\beta$ is given in Proposition 18 and $S_{K}$ is defined in formula (1.17) (see also Lemma $94 b)$ ).

Proof. We observe that, for any $P \in \mathcal{P}$, the function $t \mapsto\|P(t)\|_{X_{0}}$ is continuous in $[0,1]$, that $\|P(0)\|_{X_{0}}=\|0\|_{X_{0}}=0<\rho$, and that $\|P(1)\|_{X_{0}}=\|e\|_{X_{0}}>\rho$, with $\rho$ as in Proposition 18. Accordingly, there exists $\bar{t} \in(0,1)$ such that $\|P(\bar{t})\|_{X_{0}}=\rho$. Thus,

$$
\sup _{v \in P([0,1])} \mathcal{J}_{K, \lambda}(v) \geqslant \mathcal{J}_{K, \lambda}(P(\bar{t})) \geqslant \inf _{\substack{v \in X_{0} \\\|v\|_{X_{0}=\rho}}} \mathcal{J}_{K, \lambda}(v) .
$$

As a consequence $c \geqslant \beta$, where $\beta$ is given in Proposition 18 . 
Now, we recall that $e=\zeta_{0} u_{0}$, thanks to (3.3), and so the map $[0,1] \ni t \mapsto t \zeta_{0} u_{0}$ belongs to $\mathcal{P}$. Hence, by assumption (1.25), we conclude that

$$
\inf _{P \in \mathcal{P}} \sup _{v \in P([0,1])} \mathcal{J}_{K, \lambda}(v) \leqslant \sup _{\zeta \geqslant 0} \mathcal{J}_{K, \lambda}\left(\zeta u_{0}\right)<\frac{s}{n} S_{K}^{n /(2 s)}
$$

so that

$$
c<\frac{s}{n} S_{K}^{n /(2 s)}
$$

Hence, Claim 1 is proved.

By [3, Theorem 2.2] there exists a sequence $u_{j}$ in $X_{0}$ such that

$$
\mathcal{J}_{K, \lambda}\left(u_{j}\right) \rightarrow c
$$

and

$$
\sup \left\{\left|\left\langle\mathcal{J}_{K, \lambda}^{\prime}\left(u_{j}\right), \varphi\right\rangle\right|: \varphi \in X_{0},\|\varphi\|_{X_{0}}=1\right\} \rightarrow 0
$$

as $j \rightarrow+\infty$. Now we prove some properties of the sequence $u_{j}$.

Claim 2. The sequence $u_{j}$ is bounded in $X_{0}$.

Proof. For any $j \in \mathbb{N}$, by (3.6) and (3.7) it easily follows that there exists $\kappa>0$ such that

$$
\left|\mathcal{J}_{K, \lambda}\left(u_{j}\right)\right| \leqslant \kappa
$$

and

$$
\left|\left\langle\mathcal{J}_{K, \lambda}^{\prime}\left(u_{j}\right), \frac{u_{j}}{\left\|u_{j}\right\|_{X_{0}}}\right\rangle\right| \leqslant \kappa
$$

As a consequence of (3.8) and (3.9) we have

$$
\mathcal{J}_{K, \lambda}\left(u_{j}\right)-\frac{1}{2}\left\langle\mathcal{J}_{K, \lambda}^{\prime}\left(u_{j}\right), u_{j}\right\rangle \leqslant \kappa\left(1+\left\|u_{j}\right\|_{X_{0}}\right) .
$$

By Hölder's inequality, Lemma 8-a) and (1.8)

$$
\int_{\Omega}\left|u_{j}(x)\right| d x \leqslant|\Omega|^{\left(2^{*}-1\right) / 2^{*}}\left(\int_{\Omega}\left|u_{j}(x)\right|^{2^{*}} d x\right)^{1 / 2^{*}} \leqslant \widetilde{\kappa}|\Omega|^{\left(2^{*}-1\right) / 2^{*}}\left\|u_{j}\right\|_{X_{0}}
$$

for some positive constant $\widetilde{\kappa}$. Accordingly, by Lemma 5 and (3.11) we obtain

$$
\begin{aligned}
\mathcal{J}_{K, \lambda}\left(u_{j}\right) & -\frac{1}{2}\left\langle\mathcal{J}_{K, \lambda}^{\prime}\left(u_{j}\right), u_{j}\right\rangle \\
=- & \left(\frac{1}{2^{*}}-\frac{1}{2}\right)\left\|u_{j}\right\|_{L^{2^{*}(\Omega)}}^{2^{*}}-\int_{\Omega} F\left(x, u_{j}(x)\right) d x \\
& +\frac{1}{2} \int_{\Omega} f\left(x, u_{j}(x)\right) u_{j}(x) d x \\
\geqslant & \frac{s}{n}\left\|u_{j}\right\|_{L^{2^{*}(\Omega)}}^{2^{*}}-\varepsilon\left(1+\frac{2^{*}}{2}\right)\left\|u_{j}\right\|_{L^{2^{*}}(\Omega)}^{2^{*}}-\frac{3 M(\varepsilon)}{2} \int_{\Omega}\left|u_{j}(x)\right| d x \\
\geqslant & \frac{s}{n}\left\|u_{j}\right\|_{L^{2^{*}(\Omega)}}^{2^{*}}-\varepsilon\left(1+\frac{2^{*}}{2}\right)\left\|u_{j}\right\|_{L^{2^{*}(\Omega)}}^{2^{*}}-C_{\varepsilon}\left\|u_{j}\right\|_{X_{0}},
\end{aligned}
$$

for a suitable $C_{\varepsilon}>0$, possibly depending on $|\Omega|$. Choosing $\varepsilon>0$ small enough (i.e. 
$\varepsilon$ such that $\left.\frac{s}{n}>\varepsilon\left(1+\frac{2^{*}}{2}\right)\right),(3.10)$ and (3.12) imply that, for any $j \in \mathbb{N}$,

$$
\left\|u_{j}\right\|_{L^{2^{*}}(\Omega)}^{2^{*}} \leqslant \kappa_{*}\left(1+\left\|u_{j}\right\|_{X_{0}}\right)
$$

for a suitable positive constant $\kappa_{*}$.

Finally, by Lemma 12 (which holds true, since $0<\lambda<\lambda_{1}$ ) and Lemma 5 (used here with $\varepsilon=1$ ) it follows that

$$
\begin{aligned}
\mathcal{J}_{K, \lambda}\left(u_{j}\right) \geqslant & \frac{1}{2}\left(1-\frac{\lambda}{\lambda_{1}}\right)\left\|u_{j}\right\|_{X_{0}}^{2}-\frac{1}{2^{*}}\left\|u_{j}\right\|_{L^{2^{*}}(\Omega)}^{2^{*}}-\int_{\Omega} F\left(x, u_{j}(x)\right) d x \\
\geqslant & \frac{1}{2}\left(1-\frac{\lambda}{\lambda_{1}}\right)\left\|u_{j}\right\|_{X_{0}}^{2}-\left(\frac{1}{2^{*}}+1\right)\left\|u_{j}\right\|_{L^{2^{*}}(\Omega)}^{2^{*}} \\
& -M(1) \int_{\Omega}\left|u_{j}(x)\right| d x .
\end{aligned}
$$

Combining (3.8), (3.13) and (3.14) we conclude that

$$
\begin{aligned}
\kappa \geqslant \mathcal{J}_{K, \lambda}\left(u_{j}\right) \geqslant & \frac{1}{2}\left(1-\frac{\lambda}{\lambda_{1}}\right)\left\|u_{j}\right\|_{X_{0}}^{2}-\left(\frac{1}{2^{*}}+1\right)\left\|u_{j}\right\|_{L^{2^{*}}(\Omega)}^{2^{*}}-M(1) \int_{\Omega}\left|u_{j}(x)\right| d x \\
\geqslant & \frac{1}{2}\left(1-\frac{\lambda}{\lambda_{1}}\right)\left\|u_{j}\right\|_{X_{0}}^{2}-\kappa_{*}\left(\frac{1}{2^{*}}+1\right)\left(1+\left\|u_{j}\right\|_{X_{0}}\right) \\
& -M(1) \int_{\Omega}\left|u_{j}(x)\right| d x,
\end{aligned}
$$

so that, recalling (3.11), we see that, for any $j \in \mathbb{N}$,

$$
\left\|u_{j}\right\|_{X_{0}}^{2} \leqslant \kappa_{* *}\left(1+\left\|u_{j}\right\|_{X_{0}}\right)
$$

for a suitable positive constant $\kappa_{* *}$. Hence, the proof of Claim 2 is complete.

Claim 3. Problem (1.13) admits a solution $u_{\infty} \in X_{0}$.

Proof. Since $u_{j}$ is bounded in $X_{0}$ (thanks to Claim 2) and $X_{0}$ is a reflexive space (being a Hilbert space, by Lemma 8 8 )), up to a subsequence, still denoted by $u_{j}$, there exists $u_{\infty} \in X_{0}$ such that $u_{j} \rightarrow u_{\infty}$ weakly in $X_{0}$, that is,

$$
\begin{aligned}
\int_{\mathbb{R}^{2 n}} & \left(u_{j}(x)-u_{j}(y)\right)(\varphi(x)-\varphi(y)) K(x-y) d x d y \\
& \rightarrow \int_{\mathbb{R}^{2 n}}\left(u_{\infty}(x)-u_{\infty}(y)\right)(\varphi(x)-\varphi(y)) K(x-y) d x d y
\end{aligned}
$$

for any $\varphi \in X_{0}$ as $j \rightarrow+\infty$. Moreover, since $u_{j}$ is bounded in $X_{0}$, we deduce from (3.13) that

$$
u_{j} \text { is bounded in } L^{2^{*}}(\Omega) \text {. }
$$

Consequently, by Lemma 9 -b) and the fact that $L^{2^{*}}\left(\mathbb{R}^{n}\right)$ is a reflexive space we have that, up to a subsequence,

$$
u_{j} \rightarrow u_{\infty} \quad \text { weakly in } L^{2^{*}}\left(\mathbb{R}^{n}\right)
$$

as $j \rightarrow+\infty$, while by Lemma 9-a), up to a subsequence,

$$
\begin{aligned}
& u_{j} \rightarrow u_{\infty} \quad \text { in } L^{\nu}\left(\mathbb{R}^{n}\right), \\
& u_{j} \rightarrow u_{\infty} \quad \text { a.e. in } \mathbb{R}^{n}
\end{aligned}
$$

as $j \rightarrow+\infty$ for any $\nu \in\left[1,2^{*}\right)$ (see, for instance, [2, Theorem IV.9]). 
By (3.17) and the fact that $\left|u_{j}\right|^{2^{*}-2} u_{j}$ is bounded in $L^{2^{*} /\left(2^{*}-1\right)}(\Omega)$ we see that

$$
\left|u_{j}\right|^{2^{*}-2} u_{j} \rightarrow\left|u_{\infty}\right|^{2^{*}-2} u_{\infty} \quad \text { weakly in } L^{2^{*} /\left(2^{*}-1\right)}(\Omega)
$$

as $j \rightarrow+\infty$.

Furthermore, by (3.16) and Lemma 5 (see formula (2.10) and the fact that $\Omega$ is bounded we get that

$$
f\left(\cdot, u_{j}(\cdot)\right) \text { is bounded in } L^{2^{*} /\left(2^{*}-1\right)}(\Omega) .
$$

Moreover, by (3.19) and by the fact that the map $t \mapsto f(\cdot, t)$ is continuous in $t \in \mathbb{R}$, we get

$$
f\left(\cdot, u_{j}(\cdot)\right) \rightarrow f\left(\cdot, u_{\infty}(\cdot)\right) \text { a.e. in } \Omega
$$

as $j \rightarrow+\infty$. Then, (3.21) and (3.22) yield that

$$
f\left(\cdot, u_{j}(\cdot)\right) \rightarrow f\left(\cdot, u_{\infty}(\cdot)\right) \quad \text { weakly in } \quad L^{2^{*} /\left(2^{*}-1\right)}(\Omega)
$$

as $j \rightarrow+\infty$. As a consequence of this, since the dual of $L^{2^{*} /\left(2^{*}-1\right)}(\Omega)$ is $L^{2^{*}}(\Omega)$, it is easily seen that

$$
\int_{\Omega} f\left(x, u_{j}(x)\right) \varphi(x) d x \rightarrow \int_{\Omega} f\left(x, u_{\infty}(x)\right) \varphi(x) d x \quad \text { for any } \varphi \in L^{2^{*}}(\Omega),
$$

and so, in particular,

$$
\int_{\Omega} f\left(x, u_{j}(x)\right) \varphi(x) d x \rightarrow \int_{\Omega} f\left(x, u_{\infty}(x)\right) \varphi(x) d x \quad \text { for any } \quad \varphi \in X_{0}
$$

as $j \rightarrow+\infty$ (here we use Lemma 9 $b$ )).

Since (3.7) holds true, for any $\varphi \in X_{0}$

$$
\begin{aligned}
0 \leftarrow\left\langle\mathcal{J}_{K, \lambda}^{\prime}\left(u_{j}\right), \varphi\right\rangle= & \int_{\mathbb{R}^{2 n}}\left(u_{j}(x)-u_{j}(y)\right)(\varphi(x)-\varphi(y)) K(x-y) d x d y \\
& -\lambda \int_{\Omega} u_{j}(x) \varphi(x) d x \\
& -\int_{\Omega}\left|u_{j}(x)\right|^{2^{*}-2} u_{j}(x) \varphi(x) d x-\int_{\Omega} f\left(x, u_{j}(x)\right) \varphi(x) d x,
\end{aligned}
$$

so that, passing to the limit in this expression as $j \rightarrow+\infty$ and taking into account (3.15), (3.18), (3.20) and (3.23) we get

$$
\begin{aligned}
\int_{\mathbb{R}^{2 n}}\left(u_{\infty}(x)-u_{\infty}(y)\right)(\varphi(x)-\varphi(y)) K(x-y) d x d y-\lambda \int_{\Omega} u_{\infty}(x) \varphi(x) d x \\
-\int_{\Omega}\left|u_{\infty}(x)\right|^{2^{*}-2} u_{\infty}(x) \varphi(x) d x-\int_{\Omega} f\left(x, u_{\infty}(x)\right) \varphi(x) d x=0
\end{aligned}
$$

for any $\varphi \in X_{0}$, that is, $u_{\infty}$ is a solution of problem (1.26) and Claim 3 follows.

Claim 4. The solution $u_{\infty}$ is non-trivial, i.e. $u_{\infty} \not \equiv 0$ in $\Omega$.

Proof. Suppose, by contradiction, that $u_{\infty} \equiv 0$ in $\Omega$ (and so $u_{\infty} \equiv 0$ in $\mathbb{R}^{n}$, being $\left.u_{\infty} \in X_{0}\right)$.

Using Lemma [5] and (3.16), we see that for any $\varepsilon>0$ and $j \in \mathbb{N}$

$$
\begin{aligned}
\left|\int_{\Omega} f\left(x, u_{j}(x)\right) u_{j}(x) d x\right| & \leqslant 2^{*} \varepsilon \int_{\Omega}\left|u_{j}(x)\right|^{2^{*}} d x+M(\varepsilon) \int_{\Omega}\left|u_{j}(x)\right| d x \\
& \leqslant 2^{*} \varepsilon C+M(\varepsilon)\left\|u_{j}\right\|_{L^{1}(\Omega)}
\end{aligned}
$$


and

$$
\begin{aligned}
\left|\int_{\Omega} F\left(x, u_{j}(x)\right) d x\right| & \leqslant \varepsilon \int_{\Omega}\left|u_{j}(x)\right|^{2^{*}} d x+M(\varepsilon) \int_{\Omega}\left|u_{j}(x)\right| d x \\
& \leqslant \varepsilon C+M(\varepsilon)\left\|u_{j}\right\|_{L^{1}(\Omega)} .
\end{aligned}
$$

Keeping $\varepsilon$ fixed and taking the limit in $j$, we obtain

$$
\begin{aligned}
& \limsup _{j \rightarrow+\infty}\left|\int_{\Omega} f\left(x, u_{j}(x)\right) u_{j}(x) d x\right| \leqslant 2^{*} \varepsilon C \\
& \text { and } \quad \limsup _{j \rightarrow+\infty}\left|\int_{\Omega} F\left(x, u_{j}(x)\right) d x\right| \leqslant \varepsilon C,
\end{aligned}
$$

due to (3.18). Hence, by sending $\varepsilon \rightarrow 0^{+}$, we obtain

$$
\int_{\Omega} f\left(x, u_{j}(x)\right) u_{j}(x) d x \rightarrow 0
$$

and

$$
\int_{\Omega} F\left(x, u_{j}(x)\right) d x \rightarrow 0
$$

as $j \rightarrow+\infty$.

Moreover, being $u_{j}$ bounded in $X_{0}$ by Claim 2, from (3.7) it follows that

$$
\begin{array}{r}
0 \leftarrow\left\langle\mathcal{J}_{K, \lambda}^{\prime}\left(u_{j}\right), u_{j}\right\rangle=\int_{\mathbb{R}^{2 n}}\left|u_{j}(x)-u_{j}(y)\right|^{2} K(x-y) d x d y-\lambda \int_{\Omega}\left|u_{j}(x)\right|^{2} d x \\
-\int_{\Omega}\left|u_{j}(x)\right|^{2^{*}} d x-\int_{\Omega} f\left(x, u_{j}(x)\right) u_{j}(x) d x,
\end{array}
$$

which, thanks to (3.18) and (3.26), gives

$$
\int_{\mathbb{R}^{2 n}}\left|u_{j}(x)-u_{j}(y)\right|^{2} K(x-y) d x d y-\int_{\Omega}\left|u_{j}(x)\right|^{2^{*}} d x \rightarrow 0
$$

as $j \rightarrow+\infty$.

Now, by Claim 2 the sequence $\left\|u_{j}\right\|_{X_{0}}$ is bounded in $\mathbb{R}$. Hence, up to a subsequence, if necessary, we can assume that

$$
\left\|u_{j}\right\|_{X_{0}}^{2}=\int_{\mathbb{R}^{2 n}}\left|u_{j}(x)-u_{j}(y)\right|^{2} K(x-y) d x d y \rightarrow L
$$

and so, as a consequence of (3.28),

$$
\int_{\Omega}\left|u_{j}(x)\right|^{2^{*}} d x \rightarrow L
$$

as $j \rightarrow+\infty$. Of course $L \in[0,+\infty)$. Furthermore, by (3.6) we have that, as $j \rightarrow+\infty$,

$$
\begin{aligned}
\frac{1}{2} \int_{\mathbb{R}^{2 n}}\left|u_{j}(x)-u_{j}(y)\right|^{2} K( & x-y) d x d y-\frac{\lambda}{2} \int_{\Omega}\left|u_{j}(x)\right|^{2} d x \\
& -\frac{1}{2^{*}} \int_{\Omega}\left|u_{j}(x)\right|^{2^{*}} d x-\int_{\Omega} F\left(x, u_{j}(x)\right) d x \rightarrow c,
\end{aligned}
$$

so that, using (3.18) with $u_{\infty} \equiv 0$ in $\mathbb{R}^{n}$, (3.27), (3.29) and (3.30), it follows that

$$
c=\left(\frac{1}{2}-\frac{1}{2^{*}}\right) L=\frac{s}{n} L .
$$

Since $c \geqslant \beta>0$ by Claim 1, it is easily seen that $L>0$. 
Moreover, by Lemma 94b) and (1.17)

$$
\int_{\mathbb{R}^{2 n}}\left|u_{j}(x)-u_{j}(y)\right|^{2} K(x-y) d x d y \geqslant S_{K}\left\|u_{j}\right\|_{L^{2^{*}}(\Omega)}^{2},
$$

so that, passing to the limit as $j \rightarrow+\infty$, and taking into account (3.29) and (3.30), we get

$$
L \geqslant S_{K} L^{2 / 2^{*}},
$$

which, combined with (3.31) gives

$$
c \geqslant \frac{s}{n} S_{K}^{2^{*} /\left(2^{*}-2\right)}=\frac{s}{n} S_{K}^{n /(2 s)} .
$$

This contradicts Claim 1. Hence, $u_{\infty} \not \equiv 0$ in $\Omega$, and this ends the proof of Claim 4.

The proof of Theorem 1 is complete.

3.2. The critical case $\mathcal{L}_{K} u+\lambda u+|u|^{2^{*}-2} u=0$. In this subsection we consider problem (1.13) with $f \equiv 0$; that is, we study (1.26) and we prove Theorem 2

In order to do this it is enough to show that, when $f \equiv 0$, conditions (1.25) and (1.27) are equivalent. For this we need the following proposition:

Proposition 20. For any $u_{0} \in X_{0} \backslash\{0\}$,

$$
\begin{aligned}
\sup _{\zeta \geqslant 0}\left(\frac{\zeta^{2}}{2} \int_{\mathbb{R}^{2 n}}\left|u_{0}(x)-u_{0}(y)\right|^{2} K(\right. & x-y) d x d y-\frac{\zeta^{2} \lambda}{2} \int_{\Omega}\left|u_{0}(x)\right|^{2} d x \\
& \left.-\frac{\zeta^{2^{*}}}{2^{*}} \int_{\Omega}\left|u_{0}(x)\right|^{2^{*}} d x\right)=\frac{s}{n} S_{K, \lambda}^{n /(2 s)}\left(u_{0}\right),
\end{aligned}
$$

where the function $X_{0} \backslash\{0\} \ni v \mapsto S_{K, \lambda}(v)$ is defined in (1.20).

Proof. Let $g:[0,+\infty) \rightarrow \mathbb{R}$ be the following function:

$$
\begin{aligned}
g(\zeta)= & \frac{\zeta^{2}}{2} \int_{\mathbb{R}^{2 n}}\left|u_{0}(x)-u_{0}(y)\right|^{2} K(x-y) d x d y \\
& -\frac{\zeta^{2} \lambda}{2} \int_{\Omega}\left|u_{0}(x)\right|^{2} d x-\frac{\zeta^{2^{*}}}{2^{*}} \int_{\Omega}\left|u_{0}(x)\right|^{2^{*}} d x .
\end{aligned}
$$

Note that $g$ is differentiable in $(0,+\infty)$ and

$$
\begin{aligned}
g^{\prime}(\zeta)= & \zeta \int_{\mathbb{R}^{2 n}}\left|u_{0}(x)-u_{0}(y)\right|^{2} K(x-y) d x d y \\
& -\zeta \lambda \int_{\Omega}\left|u_{0}(x)\right|^{2} d x-\zeta^{2^{*}-1} \int_{\Omega}\left|u_{0}(x)\right|^{2^{*}} d x,
\end{aligned}
$$

so that $g^{\prime}(\zeta) \geqslant 0$ if and only if

$$
\zeta \leqslant \bar{\zeta}=\left(\frac{\int_{\mathbb{R}^{2 n}}\left|u_{0}(x)-u_{0}(y)\right|^{2} K(x-y) d x d y-\lambda \int_{\Omega}\left|u_{0}(x)\right|^{2} d x}{\int_{\Omega}\left|u_{0}(x)\right|^{2^{*}} d x}\right)^{1 /\left(2^{*}-2\right)} .
$$

Therefore $\bar{\zeta}$ is a maximum for $g$ and

$$
\sup _{\zeta \geqslant 0} g(\zeta)=\max _{\zeta \geqslant 0} g(\zeta)=g(\bar{\zeta})=\frac{s}{n} S_{K, \lambda}^{n /(2 s)}\left(u_{0}\right) .
$$

This concludes the proof of Proposition 20 . 
3.2.1. End of the proof of Theorem 2, By Proposition 20 we deduce that, when $f \equiv 0$ in $\Omega \times \mathbb{R}$, condition (1.25) reads as follows:

$$
\begin{aligned}
& \text { there exists } u_{0} \in X_{0} \backslash\{0\} \text { with } u_{0} \geqslant 0 \text { a.e. in } \mathbb{R}^{n}, \\
& \text { such that } S_{K, \lambda}\left(u_{0}\right)<S_{K} .
\end{aligned}
$$

It is easily seen that conditions (3.33) and (1.27) are equivalent. Indeed, suppose that (3.33) holds true. Then,

$$
S_{K, \lambda}:=\inf _{v \in X_{0} \backslash\{0\}} S_{K, \lambda}(v) \leqslant S_{K, \lambda}\left(u_{0}\right)<S_{K},
$$

while, if (1.27) is valid, then there exists $\widetilde{v} \in X_{0} \backslash\{0\}$ such that

$$
S_{K, \lambda}(\widetilde{v})<S_{K}
$$

Thus, condition (3.33) follows by taking $u_{0}=|\widetilde{v}| \in X_{0}$. We remark that this choice is possible since for any $v \in X_{0}$ its positive part $v^{+}=\max \{v, 0\}$ and its negative part $v^{-}=\max \{-v, 0\}$ belong to $X_{0}$ too, by [13, Lemma 5.2] (hence, $|v|=v^{+}+v^{-} \in X_{0}$, being $X_{0}$ a linear space). Indeed, by triangle inequality, a.e. $x, y \in \mathbb{R}^{n}$

$$
|| \widetilde{v}(x)|-| \widetilde{v}(y)|| \leqslant|\widetilde{v}(x)-\widetilde{v}(y)|
$$

This and (3.35) give

$$
S_{K, \lambda}\left(u_{0}\right)=S_{K, \lambda}(|\widetilde{v}|) \leqslant S_{K, \lambda}(\widetilde{v})<S_{K} .
$$

Hence, conditions (3.33) and (1.27) are equivalent, and so Theorem 2 comes from Theorem 1 .

\section{A critical fractional Laplace equation}

The aim of this section is to consider the particular fractional kernel

$$
K(x)=|x|^{-(n+2 s)},
$$

which gives rise to the fractional Laplace operator $-(-\Delta)^{s}$ defined in (1.3). Hence, here we study

$$
\begin{cases}(-\Delta)^{s} u-\lambda u=|u|^{2^{*}-2} u+f(x, u) & \text { in } \Omega, \\ u=0 & \text { in } \mathbb{R}^{n} \backslash \Omega,\end{cases}
$$

which reduces, when $f \equiv 0$ in $\Omega \times \mathbb{R}$, to problem (1.2). More precisely, we are interested in the weak formulation of these problems, which is given by (1.28) and (1.30), respectively.

4.1. The critical case for $(-\Delta)^{s}$ with a lower order perturbation: Proof of Theorem [3. In order to prove Theorem 3 it is enough to note that, in this setting, condition (1.29) implies (1.25), thanks to Lemma 7. $b$ ) and Remark 11.

Hence, Theorem 3 follows from Theorem 1 and from the fact that $X_{0} \subseteq H^{s}\left(\mathbb{R}^{n}\right)$ by Lemma 7 f $a$ ) (alternatively, we can use Lemma 7f $b$ ), since the solution $u$ provided by Theorem 1 is such that $u=0$ a.e. in $\mathbb{R}^{n} \backslash \Omega$ ). 
4.2. The critical case $(-\Delta)^{s} u-\lambda u=|u|^{2^{*}-2} u$ : Proof of Theorem 4, The idea consists of applying Theorem 3 with $f \equiv 0$ in $\Omega \times \mathbb{R}$.

First of all, note that by Proposition 20 with $K(x)=|x|^{-(n+2 s)}-$ see also Lemma (7) $b$ ) - we have that if $u_{0} \in H^{s}\left(\mathbb{R}^{n}\right) \backslash\{0\}$, with $u_{0}=0$ a.e. outside $\Omega$, then

$$
\begin{aligned}
\sup _{\zeta \geqslant 0}\left(\frac{\zeta^{2}}{2} \int_{\mathbb{R}^{2 n}} \frac{\left|u_{0}(x)-u_{0}(y)\right|^{2}}{|x-y|^{+2 s}} d x\right. & d y-\frac{\zeta^{2} \lambda}{2} \int_{\Omega}\left|u_{0}(x)\right|^{2} d x \\
& \left.-\frac{\zeta^{2^{*}}}{2^{*}} \int_{\Omega}\left|u_{0}(x)\right|^{2^{*}} d x\right)=\frac{s}{n} S_{s, \lambda}^{n /(2 s)}\left(u_{0}\right)
\end{aligned}
$$

where the function $H^{s}\left(\mathbb{R}^{n}\right) \backslash\{0\} \ni v \mapsto S_{s, \lambda}(v)$ is defined in (1.24).

Therefore, when $f \equiv 0$ in $\Omega \times \mathbb{R}$, condition (1.29) reads as follows:

there exists $u_{0} \in H^{s}\left(\mathbb{R}^{n}\right) \backslash\{0\}$ with $u_{0} \geqslant 0$ a.e. in $\mathbb{R}^{n}$ and $u_{0}=0$ a.e. in $\mathbb{R}^{n} \backslash \Omega$,

$$
\text { such that } S_{s, \lambda}\left(u_{0}\right)<S_{s} \text {. }
$$

Hence, in order to prove Theorem 4 it is enough to show that condition (4.2) is satisfied for some appropriate function $u_{0}$. For this, we proceed again by steps.

Claim 5. The infimum in formula (1.21) is attained; that is,

$$
S_{s}=S_{s}(\widetilde{u})
$$

where

$$
\widetilde{u}(x)=\kappa\left(\mu^{2}+\left|x-x_{0}\right|^{2}\right)^{-(n-2 s) / 2}, \quad x \in \mathbb{R}^{n},
$$

with $\kappa \in \mathbb{R} \backslash\{0\}, \mu>0$ and $x_{0} \in \mathbb{R}^{n}$ fixed constants.

Equivalently, the function $\bar{u}$ defined as

$$
\bar{u}(x)=\frac{\widetilde{u}(x)}{\|\widetilde{u}\|_{L^{2^{*}}\left(\mathbb{R}^{n}\right)}}
$$

is such that

$$
S_{s}=\inf _{\substack{v \in H^{s}\left(\mathbb{R}^{n}\right) \\\|v\|_{L^{2 *}\left(\mathbb{R}^{n}\right)}=1}} \int_{\mathbb{R}^{2 n}} \frac{|v(x)-v(y)|^{2}}{|x-y|^{n+2 s}} d x d y=\int_{\mathbb{R}^{2 n}} \frac{|\bar{u}(x)-\bar{u}(y)|^{2}}{|x-y|^{n+2 s}} d x d y .
$$

Proof. This assertion comes from [6, Theorem 1.1].

As in the classical case of the Laplacian, starting from $\bar{u}$ we can construct an explicit solution of the limiting problem

$$
(-\Delta)^{s} u=|u|^{2^{*}-2} u \quad \text { in } \mathbb{R}^{n}
$$

as we show in the following result:

Claim 6. The function

$$
u^{*}(x)=\bar{u}\left(\frac{x}{S_{s}^{1 /(2 s)}}\right), \quad x \in \mathbb{R}^{n},
$$

is a solution of problem (4.6) satisfying the property

$$
\left\|u^{*}\right\|_{L^{2^{*}\left(\mathbb{R}^{n}\right)}}^{2^{*}}=S_{s}^{n /(2 s)} .
$$


Proof. By Claim [5 there exists a Lagrange multiplier $\gamma \in \mathbb{R}$ such that

$$
\int_{\mathbb{R}^{2 n}} \frac{(\bar{u}(x)-\bar{u}(y))(\varphi(x)-\varphi(y))}{|x-y|^{n+2 s}} d x d y=\gamma \int_{\mathbb{R}^{n}}|\bar{u}(x)|^{2^{*}-2} \bar{u}(x) \varphi(x) d x
$$

for any $\varphi \in H^{s}\left(\mathbb{R}^{n}\right)$.

Taking $\varphi=\bar{u}$ as a test function in (4.9) and again using Claim 5 we get

$$
S_{s}=\int_{\mathbb{R}^{2 n}} \frac{|\bar{u}(x)-\bar{u}(y)|^{2}}{|x-y|^{n+2 s}} d x d y=\gamma
$$

since $\|\bar{u}\|_{L^{2^{*}\left(\mathbb{R}^{n}\right)}}=1$ by construction (see (4.4)).

Let $u^{*}$ be the function defined as in (4.7). Note that $u^{*} \in H^{s}\left(\mathbb{R}^{n}\right) \backslash\{0\}$. Moreover, for any $\varphi \in H^{s}\left(\mathbb{R}^{n}\right)$, by (4.9) and (4.10) we have

$$
\begin{aligned}
\int_{\mathbb{R}^{2 n}} \frac{\left(u^{*}(x)-u^{*}(y)\right)(\varphi(x)-\varphi(y))}{|x-y|^{n+2 s}} d x d y \\
\quad=S_{s}^{(n-2 s) /(2 s)} \int_{\mathbb{R}^{2 n}} \frac{(\bar{u}(x)-\bar{u}(y))\left(\varphi\left(S_{s}^{1 /(2 s)} x\right)-\varphi\left(S_{s}^{1 /(2 s)} y\right)\right)}{|x-y|^{n+2 s}} d x d y \\
\quad=S_{s}^{n /(2 s)} \int_{\mathbb{R}^{n}}|\bar{u}(x)|^{2^{*}-2} \bar{u}(x) \varphi\left(S_{s}^{1 /(2 s)} x\right) d x \\
\quad=\int_{\mathbb{R}^{n}}\left|u^{*}(x)\right|^{2^{*}-2} u^{*}(x) \varphi(x) d x
\end{aligned}
$$

that is, $u^{*}$ is a solution of problem (4.6).

Finally, taking $\varphi=u^{*}$ as a test function in (4.11) and using (4.4) and (4.7) we get

$$
\int_{\mathbb{R}^{2 n}} \frac{\left|u^{*}(x)-u^{*}(y)\right|^{2}}{|x-y|^{n+2 s}} d x d y=\int_{\mathbb{R}^{n}}\left|u^{*}(x)\right|^{2^{*}} d x=S_{s}^{n /(2 s)} \int_{\mathbb{R}^{n}}|\bar{u}(x)|^{2^{*}} d x=S_{s}^{n /(2 s)},
$$

so that the proof of Claim 6 is complete.

Now, we consider the family of functions $U_{\varepsilon}$ defined as

$$
U_{\varepsilon}(x)=\varepsilon^{-(n-2 s) / 2} u^{*}(x / \varepsilon), \quad x \in \mathbb{R}^{n},
$$

for any $\varepsilon>0$. The scaling invariance of the problem is pointed out in the following observation:

Claim 7. The function $U_{\varepsilon}$ is a solution of problem (4.6) and verifies the following equalities:

$$
\int_{\mathbb{R}^{2 n}} \frac{\left|U_{\varepsilon}(x)-U_{\varepsilon}(y)\right|^{2}}{|x-y|^{n+2 s}} d x d y=\int_{\mathbb{R}^{n}}\left|U_{\varepsilon}(x)\right|^{2^{*}} d x=S_{s}^{n /(2 s)}
$$

for any $\varepsilon>0$. 
Proof. By (4.12) and Claim 6 it easily follows that $U_{\varepsilon} \in H^{s}\left(\mathbb{R}^{n}\right)$ and

$$
\begin{aligned}
\int_{\mathbb{R}^{2 n}} \frac{\left(U_{\varepsilon}(x)-\right.}{\left.U_{\varepsilon}(y)\right)(\varphi(x)-\varphi(y))} d x d y & |x-y|^{n+2 s} \\
= & \varepsilon^{(n-2 s) / 2} \int_{\mathbb{R}^{2 n}} \frac{\left(u^{*}(x)-u^{*}(y)\right)(\varphi(\varepsilon x)-\varphi(\varepsilon y))}{|x-y|^{n+2 s}} d x d y \\
& =\varepsilon^{(n-2 s) / 2} \int_{\mathbb{R}^{n}}\left|u^{*}(x)\right|^{2^{*}-2} u^{*}(x) \varphi(\varepsilon x) d x \\
& =\int_{\mathbb{R}^{n}}\left|U_{\varepsilon}(x)\right|^{2^{*}-2} U_{\varepsilon}(x) \varphi(x) d x,
\end{aligned}
$$

for any $\varphi \in H^{s}\left(\mathbb{R}^{n}\right)$ and $\varepsilon>0$. Hence, $U_{\varepsilon}$ is a solution of problem (4.6) for any $\varepsilon>0$.

Taking $\varphi=U_{\varepsilon}$ in (4.14) (this choice is admissible, since $U_{\varepsilon} \in H^{s}\left(\mathbb{R}^{n}\right)$ ), we get

$$
\int_{\mathbb{R}^{2 n}} \frac{\left|U_{\varepsilon}(x)-U_{\varepsilon}(y)\right|^{2}}{|x-y|^{n+2 s}} d x d y=\int_{\mathbb{R}^{n}}\left|U_{\varepsilon}(x)\right|^{2^{*}} d x
$$

for any $\varepsilon>0$. The scale invariance of the norm in $L^{2^{*}}\left(\mathbb{R}^{n}\right)$ under the scaling $u^{*} \mapsto U_{\varepsilon}$ and (4.8) give

$$
\int_{\mathbb{R}^{n}}\left|U_{\varepsilon}(x)\right|^{2^{*}} d x=\int_{\mathbb{R}^{n}}\left|u^{*}(x)\right|^{2^{*}} d x=S_{s}^{n /(2 s)}
$$

for any $\varepsilon>0$, and so Claim 7 is proved.

In order to check (4.2), it is now necessary to appropriately put $U_{\varepsilon}$ to zero outside $\Omega$, according to the following procedure. Let us fix $\delta>0$ such that

$$
B_{4 \delta} \subset \Omega
$$

and let $\eta \in C^{\infty}\left(\mathbb{R}^{n}\right)$ be such that $0 \leqslant \eta \leqslant 1$ in $\mathbb{R}^{n}, \eta \equiv 1$ in $B_{\delta}$ and $\eta \equiv 0$ in $\mathcal{C} B_{2 \delta}$, where $B_{\delta}=B(0, \delta)$ and $\mathcal{C} B_{\delta}=\mathbb{R}^{n} \backslash B_{\delta}$. For every $\varepsilon>0$ we denote by $u_{\varepsilon}$ the following function:

$$
u_{\varepsilon}(x)=\eta(x) U_{\varepsilon}(x), \quad x \in \mathbb{R}^{n},
$$

where $U_{\varepsilon}$ is given in (4.12).

In order to prove Theorem 4 we need to check condition (4.2) for $u_{\varepsilon}$ (at least for some suitably small $\varepsilon$ ), and for this we will estimate $S_{s, \lambda}\left(u_{\varepsilon}\right)$. In the setting of the fractional Laplacian this estimate is more delicate than in the case of the Laplacian, due to the non-local nature of the operator $(-\Delta)^{s}$.

For this we need some preliminary claims. In what follows we suppose that, up to a translation, $x_{0}=0$ in (4.3).

Claim 8. Let $\varrho>0$ and $\mu$ be as in (4.3). If $x \in \mathcal{C} B_{\varrho}$, then

$$
\left|u_{\varepsilon}(x)\right| \leqslant\left|U_{\varepsilon}(x)\right| \leqslant C \varepsilon^{(n-2 s) / 2}
$$

for any $\varepsilon>0$ and for some positive constant $C$, possibly depending on $\mu, \varrho, s$ and $n$.

Proof. Using (4.12), (4.7), (4.4) and (4.3) (with $x_{0}=0$ ), we have that

$$
U_{\varepsilon}(x)=\widetilde{\kappa} \varepsilon^{-(n-2 s) / 2}\left(\mu^{2}+\left|\frac{x}{\varepsilon S_{s}^{1 /(2 s)}}\right|^{2}\right)^{-(n-2 s) / 2},
$$


for a suitable $\widetilde{\kappa} \in \mathbb{R} \backslash\{0\}$. If $x \in \mathcal{C} B_{\varrho}$, we deduce that

$$
\left|U_{\varepsilon}(x)\right| \leqslant C \varepsilon^{-(n-2 s) / 2}\left(\mu^{2}+\left(\frac{\varrho}{\varepsilon S_{s}^{1 /(2 s)}}\right)^{2}\right)^{-(n-2 s) / 2} \leqslant C \varepsilon^{(n-2 s) / 2},
$$

and so Claim 8 follows, being $\eta \leqslant 1$ in $\mathbb{R}^{n}$.

Claim 9. Let $\varrho>0$ and $\mu$ be as in (4.3). If $x \in \mathcal{C} B_{\varrho}$, then

$$
\left|\nabla u_{\varepsilon}(x)\right| \leqslant C \varepsilon^{(n-2 s) / 2}
$$

for any $\varepsilon>0$ and for some positive constant $C$, possibly depending on $\mu, \varrho, s$ and $n$.

Proof. First of all we observe that, for any $|\xi| \geqslant \varrho$, we have that

$$
\begin{aligned}
&\left(1+\left|\frac{\xi}{\varepsilon}\right|^{2}\right)^{-(n-2 s) / 2}+\frac{|\xi|}{\varepsilon^{2}}\left(1+\left|\frac{\xi}{\varepsilon}\right|^{2}\right)^{-1-(n-2 s) / 2} \\
& \leqslant\left(1+\left|\frac{\xi}{\varepsilon}\right|^{2}\right)^{-(n-2 s) / 2}+\frac{1}{\varrho}\left|\frac{\xi}{\varepsilon}\right|^{2}\left(1+\left|\frac{\xi}{\varepsilon}\right|^{2}\right)^{-1-(n-2 s) / 2} \\
& \leqslant\left(1+\frac{1}{\varrho}\right)\left(1+\left|\frac{\xi}{\varepsilon}\right|^{2}\right)^{-(n-2 s) / 2} \\
& \leqslant\left(1+\frac{1}{\varrho}\right)\left(1+\left|\frac{\varrho}{\varepsilon}\right|^{2}\right)^{-(n-2 s) / 2} \\
& \leqslant\left(1+\frac{1}{\varrho}\right)\left(\frac{\varepsilon}{\varrho}\right)^{n-2 s} .
\end{aligned}
$$

As a consequence of (4.16) and (4.20) we have that, for any $x \in \mathcal{C} B_{\varrho}$,

$$
\begin{aligned}
&\left|\nabla u_{\varepsilon}(x)\right| \leqslant C \varepsilon^{-(n-2 s) / 2}\left[\left(\mu^{2}+\left|\frac{x}{\varepsilon S_{s}^{1 /(2 s)}}\right|^{2}\right)^{-(n-2 s) / 2}\right. \\
&\left.+\frac{1}{\varepsilon S_{s}^{1 /(2 s)}}\left|\frac{x}{\varepsilon S_{s}^{1 /(2 s)}}\right|\left(\mu^{2}+\left|\frac{x}{\varepsilon S_{s}^{1 /(2 s)}}\right|^{2}\right)^{-1-(n-2 s) / 2}\right] \\
& \leqslant C \varepsilon^{-(n-2 s) / 2} \cdot \varepsilon^{n-2 s}=C \varepsilon^{(n-2 s) / 2}
\end{aligned}
$$

which proves Claim 9 .

Claim 10. Let $\delta$ be as in (4.15) and $\mu$ be as in (4.3). The following assertions hold true:

a) for any $x \in \mathbb{R}^{n}$ and $y \in \mathcal{C} B_{\delta}$, with $|x-y| \leqslant \delta / 2$,

$$
\left|u_{\varepsilon}(x)-u_{\varepsilon}(y)\right| \leqslant C \varepsilon^{(n-2 s) / 2}|x-y| ;
$$

b) for any $x, y \in \mathcal{C} B_{\delta}$

$$
\left|u_{\varepsilon}(x)-u_{\varepsilon}(y)\right| \leqslant C \varepsilon^{(n-2 s) / 2} \min \{1,|x-y|\}
$$

for any $\varepsilon>0$ and for some positive constant $C$, possibly depending on $\mu, \delta, s$ and $n$. 
Proof. Let us start by proving assertion $a$ ). For this let $x \in \mathbb{R}^{n}$ and $y \in \mathcal{C} B_{\delta}$ with $|x-y| \leqslant \delta / 2$, and let $\xi$ be any point on the segment joining $x$ and $y$. Then

$$
\xi=t x+(1-t) y, \quad \text { for some } t \in[0,1],
$$

so that

$$
|\xi|=|y+t(x-y)| \geqslant|y|-t|x-y| \geqslant \delta-t(\delta / 2) \geqslant \delta / 2 .
$$

This and Claim 9 (here used with $\varrho=\delta / 2$ ) imply that $\left|\nabla u_{\varepsilon}(\xi)\right| \leqslant C \varepsilon^{(n-2 s) / 2}$, and so, by a first order Taylor expansion,

$$
\left|u_{\varepsilon}(x)-u_{\varepsilon}(y)\right| \leqslant C \varepsilon^{(n-2 s) / 2}|x-y|,
$$

which proves (4.21).

Now, we show $b)$. For this let $x, y \in \mathcal{C} B_{\delta}$. If $|x-y| \leqslant \delta / 2$, then $b$ ) follows from $a)$, so we may suppose $|x-y|>\delta / 2$. Then

$$
\left|u_{\varepsilon}(x)-u_{\varepsilon}(y)\right| \leqslant\left|u_{\varepsilon}(x)\right|+\left|u_{\varepsilon}(y)\right| \leqslant C \varepsilon^{(n-2 s) / 2},
$$

thanks to Claim 8 (here used with $\varrho=\delta$ ), and this completes the proof of (4.22).

Now we can estimate the Gagliardo seminorm of $u_{\varepsilon}$ according to the following result:

Proposition 21. Let $s \in(0,1)$ and $n>2 s$. Then, the following estimate holds true:

as $\varepsilon \rightarrow 0$.

$$
\int_{\mathbb{R}^{2 n}} \frac{\left|u_{\varepsilon}(x)-u_{\varepsilon}(y)\right|^{2}}{|x-y|^{n+2 s}} d x d y \leqslant S_{s}^{n /(2 s)}+\mathcal{O}\left(\varepsilon^{n-2 s}\right)
$$

Proof. The proof makes use of the previous estimates and it is a bit complicated definitely more difficult than the one for similar results in the case of the Laplacian. The additional complications arise not only from technical obstacles (differentiating is of course simpler than integrating), but also from a different distribution of the energy density. In particular, in the fractional case, the interaction between the interior and the exterior of the cut-off in (4.16) is small but no longer negligible (indeed it will contribute to the total energy according to the forthcoming estimate (4.27), (4.28) and (4.31)). To keep track of these non-local interactions, we introduce the notation

$$
\mathbb{D}:=\left\{(x, y) \in \mathbb{R}^{2 n}: x \in B_{\delta}, y \in \mathcal{C} B_{\delta} \text { and }|x-y|>\delta / 2\right\}
$$

and

$$
\mathbb{E}:=\left\{(x, y) \in \mathbb{R}^{2 n}: x \in B_{\delta}, y \in \mathcal{C} B_{\delta} \text { and }|x-y| \leqslant \delta / 2\right\},
$$

where $\delta$ is as in (4.15).

By (4.16) we have that

$$
\begin{aligned}
\int_{\mathbb{R}^{2 n}} \frac{\left|u_{\varepsilon}(x)-u_{\varepsilon}(y)\right|^{2}}{|x-y|^{n+2 s}} d x d y=\int_{B_{\delta} \times B_{\delta}} \frac{\left|U_{\varepsilon}(x)-U_{\varepsilon}(y)\right|^{2}}{|x-y|^{n+2 s}} d x d y \\
+2 \int_{\mathbb{D}} \frac{\left|u_{\varepsilon}(x)-u_{\varepsilon}(y)\right|^{2}}{|x-y|^{n+2 s}} d x d y \\
+2 \int_{\mathbb{E}} \frac{\left|u_{\varepsilon}(x)-u_{\varepsilon}(y)\right|^{2}}{|x-y|^{n+2 s}} d x d y \\
+\int_{\left(\mathcal{C} B_{\delta}\right) \times\left(\mathcal{C} B_{\delta}\right)} \frac{\left|u_{\varepsilon}(x)-u_{\varepsilon}(y)\right|^{2}}{|x-y|^{n+2 s}} d x d y .
\end{aligned}
$$


By (4.16) and Claim 10 (here, in particular, we use (4.22) ) we have

$$
\begin{aligned}
& \int_{\left(\mathcal{C} B_{\delta}\right) \times\left(\mathcal{C} B_{\delta}\right)} \frac{\left|u_{\varepsilon}(x)-u_{\varepsilon}(y)\right|^{2}}{|x-y|^{n+2 s}} d x d y \\
& \leqslant C \varepsilon^{n-2 s} \int_{B_{2 \delta} \times \mathbb{R}^{n}} \frac{\min \left\{1,|x-y|^{2}\right\}}{|x-y|^{n+2 s}} d x d y=\mathcal{O}\left(\varepsilon^{n-2 s}\right)
\end{aligned}
$$

while, by (4.21),

$$
\begin{aligned}
\int_{\mathbb{E}} \frac{\left|u_{\varepsilon}(x)-u_{\varepsilon}(y)\right|^{2}}{|x-y|^{n+2 s}} d x d y & \leqslant C \varepsilon^{n-2 s} \int_{\substack{x \in B_{\delta}, y \in \mathcal{C} B_{\delta} \\
|x-y| \leqslant \delta / 2}} \frac{|x-y|^{2}}{|x-y|^{n+2 s}} d x d y \\
& \leqslant C \varepsilon^{n-2 s} \int_{|x| \leqslant \delta} d x \int_{|\xi| \leqslant \delta / 2} \frac{1}{|\xi|^{n+2 s-2}} d \xi \\
& =\mathcal{O}\left(\varepsilon^{n-2 s}\right),
\end{aligned}
$$

as $\varepsilon \rightarrow 0$. In both these estimates we use that $s \in(0,1)$.

Now, in (4.23) it remains to estimate the integral on $\mathbb{D}$, that is,

$$
\int_{\mathbb{D}} \frac{\left|u_{\varepsilon}(x)-u_{\varepsilon}(y)\right|^{2}}{|x-y|^{n+2 s}} d x d y .
$$

For this, recalling that $u_{\varepsilon}(x)=U_{\varepsilon}(x)$ for any $x \in B_{\delta}$ thanks to (4.16), we note that for any $(x, y) \in \mathbb{D}$

$$
\begin{aligned}
\left|u_{\varepsilon}(x)-u_{\varepsilon}(y)\right|^{2}= & \left|U_{\varepsilon}(x)-u_{\varepsilon}(y)\right|^{2} \\
= & \left|\left(U_{\varepsilon}(x)-U_{\varepsilon}(y)\right)+\left(U_{\varepsilon}(y)-u_{\varepsilon}(y)\right)\right|^{2} \\
\leqslant & \left|U_{\varepsilon}(x)-U_{\varepsilon}(y)\right|^{2}+\left|U_{\varepsilon}(y)-u_{\varepsilon}(y)\right|^{2} \\
& +2\left|U_{\varepsilon}(x)-U_{\varepsilon}(y)\right|\left|U_{\varepsilon}(y)-u_{\varepsilon}(y)\right|,
\end{aligned}
$$

so that

$$
\begin{aligned}
\int_{\mathbb{D}} \frac{\left|u_{\varepsilon}(x)-u_{\varepsilon}(y)\right|^{2}}{|x-y|^{n+2 s}} d x d y & \\
& \leqslant \int_{\mathbb{D}} \frac{\left|U_{\varepsilon}(x)-U_{\varepsilon}(y)\right|^{2}}{|x-y|^{n+2 s}} d x d y+\int_{\mathbb{D}} \frac{\left|U_{\varepsilon}(y)-u_{\varepsilon}(y)\right|^{2}}{|x-y|^{n+2 s}} d x d y \\
& +2 \int_{\mathbb{D}} \frac{\left|U_{\varepsilon}(x)-U_{\varepsilon}(y)\right|\left|U_{\varepsilon}(y)-u_{\varepsilon}(y)\right|}{|x-y|^{n+2 s}} d x d y .
\end{aligned}
$$

Hence, in order to estimate (4.26), we bound the last two terms in the right-hand side of (4.27). 
By exploiting Claim 8 (here used with $\varrho=\delta$ ), we obtain

$$
\begin{aligned}
\int_{\mathbb{D}} \frac{\left|U_{\varepsilon}(y)-u_{\varepsilon}(y)\right|^{2}}{|x-y|^{n+2 s}} d x d y & \leqslant \int_{\mathbb{D}} \frac{\left(\left|U_{\varepsilon}(y)\right|+\left|u_{\varepsilon}(y)\right|\right)^{2}}{|x-y|^{n+2 s}} d x d y \\
& \leqslant 4 \int_{\mathbb{D}} \frac{\left|U_{\varepsilon}(y)\right|^{2}}{|x-y|^{n+2 s}} d x d y \\
& \leqslant C \varepsilon^{n-2 s} \int_{\substack{x \in B_{\delta}, y \in \mathcal{C} B_{\delta} \\
|x-y|>\delta / 2}} \frac{1}{|x-y|^{n+2 s}} d x d y \\
& \leqslant C \varepsilon^{n-2 s} \int_{|\zeta| \leqslant \delta} d \zeta \int_{|\xi|>\delta / 2} \frac{1}{|\xi|^{n+2 s}} d \xi \\
& =\mathcal{O}\left(\varepsilon^{n-2 s}\right),
\end{aligned}
$$

as $\varepsilon \rightarrow 0$.

In order to estimate the last term in the right-hand side of (4.27), first of all we note that, once more by (4.18) (which is valid for any $x \in \mathbb{R}^{n}$ ) and Claim 8 ,

$$
\left|U_{\varepsilon}(x)\right|\left|U_{\varepsilon}(y)\right| \leqslant C\left(\mu^{2}+\left|\frac{x}{\varepsilon S_{s}^{1 /(2 s)}}\right|^{2}\right)^{-(n-2 s) / 2}
$$

for any $(x, y) \in \mathbb{D}$.

Therefore, by using the shorthand notation

$$
\delta_{\varepsilon}:=\delta /\left(\varepsilon S_{s}^{1 /(2 s)}\right)
$$

and the change of variable $\zeta:=x /\left(\varepsilon S_{s}^{1 /(2 s)}\right)$ and $\xi:=x-y($ and up to renaming $C)$, we have that

$$
\begin{aligned}
\int_{\mathbb{D}} \frac{\left|U_{\varepsilon}(x)\right|\left|U_{\varepsilon}(y)\right|}{|x-y|^{n+2 s}} d x d y \leqslant C & \int_{\mathbb{D}}\left(\mu^{2}+\left|\frac{x}{\varepsilon S_{s}^{1 /(2 s)}}\right|^{2}\right)^{-(n-2 s) / 2}|x-y|^{-(n+2 s)} d x d y \\
& \leqslant C \varepsilon^{n} \int_{\substack{\zeta \in B_{\delta} \\
|\xi|>\delta / 2}}\left(\mu^{2}+|\zeta|^{2}\right)^{-(n-2 s) / 2}|\xi|^{-(n+2 s)} d \zeta d \xi \\
& \leqslant C \varepsilon^{n} \int_{\zeta \in B_{\delta_{\varepsilon}}}\left(\mu^{2}+|\zeta|^{2}\right)^{-(n-2 s) / 2} d \zeta \\
& \leqslant C \varepsilon^{n}\left[1+\int_{\zeta \in B_{\delta_{\varepsilon}} \backslash B_{1}}\left(\mu^{2}+|\zeta|^{2}\right)^{-(n-2 s) / 2} d \zeta\right] \\
& \leqslant C \varepsilon^{n}\left[1+\int_{\zeta \in B_{\delta_{\varepsilon}} \backslash B_{1}}^{\left.|\zeta|^{-(n-2 s)} d \zeta\right]}\right. \\
& =C \varepsilon^{n}\left[1+\int_{1}^{\delta /\left(\varepsilon S_{s}^{1 /(2 s)}\right)} \rho^{-(n-2 s)+(n-1)} d \rho\right] \\
& =C \varepsilon^{n}\left(1+\varepsilon^{-2 s}\right) \\
& =\mathcal{O}\left(\varepsilon^{n-2 s}\right),
\end{aligned}
$$

as $\varepsilon \rightarrow 0$. Here we again use that $s \in(0,1)$. 
As a consequence, recalling (4.16) and Claim 8, we get

$$
\begin{aligned}
\int_{\mathbb{D}} \frac{\left|U_{\varepsilon}(x)\right|\left|U_{\varepsilon}(y)-u_{\varepsilon}(y)\right|}{|x-y|^{n+2 s}} d x d y & \leqslant \int_{\mathbb{D}} \frac{\left|U_{\varepsilon}(x)\right|\left(\left|U_{\varepsilon}(y)\right|+\left|u_{\varepsilon}(y)\right|\right)}{|x-y|^{n+2 s}} d x d y \\
& \leqslant 2 \int_{\mathbb{D}} \frac{\left|U_{\varepsilon}(x)\right|\left|U_{\varepsilon}(y)\right|}{|x-y|^{n+2 s}} d x d y \\
& =\mathcal{O}\left(\varepsilon^{n-2 s}\right),
\end{aligned}
$$

as $\varepsilon \rightarrow 0$.

On the other hand, again by (4.16) and Claim 8

$$
\begin{aligned}
\int_{\mathbb{D}} \frac{\left|U_{\varepsilon}(y)\right|\left|U_{\varepsilon}(y)-u_{\varepsilon}(y)\right|}{|x-y|^{n+2 s}} d x d y & \leqslant 2 \int_{\mathbb{D}} \frac{\left|U_{\varepsilon}(y)\right|^{2}}{|x-y|^{n+2 s}} d x d y \\
& \leqslant C \varepsilon^{n-2 s} \int_{\substack{x \in B_{\delta}, y \in \mathcal{C} B_{\delta} \\
|x-y|>\delta / 2}} \frac{1}{|x-y|^{n+2 s}} d x d y \\
& =\mathcal{O}\left(\varepsilon^{n-2 s}\right),
\end{aligned}
$$

as $\varepsilon \rightarrow 0$ (here we argue as in (4.28) ). Putting together (4.29) with (4.30), we infer that

$$
\begin{aligned}
\int_{\mathbb{D}} & \frac{\left|U_{\varepsilon}(x)-U_{\varepsilon}(y)\right|\left|U_{\varepsilon}(y)-u_{\varepsilon}(y)\right|}{|x-y|^{n+2 s}} d x d y \\
& \leqslant \int_{\mathbb{D}} \frac{\left|U_{\varepsilon}(x)\right|\left|U_{\varepsilon}(y)-u_{\varepsilon}(y)\right|}{|x-y|^{n+2 s}} d x d y+\int_{\mathbb{D}} \frac{\left|U_{\varepsilon}(y)\right|\left|U_{\varepsilon}(y)-u_{\varepsilon}(y)\right|}{|x-y|^{n+2 s}} d x d y \\
\quad & \mathcal{O}\left(\varepsilon^{n-2 s}\right)
\end{aligned}
$$

as $\varepsilon \rightarrow 0$.

Finally, by (4.23)-(4.25), (4.27), (4.28) and (4.31) we get5

$$
\begin{aligned}
\int_{\mathbb{R}^{2 n}} \frac{\left|u_{\varepsilon}(x)-u_{\varepsilon}(y)\right|^{2}}{|x-y|^{n+2 s}} d x d y= & \int_{B_{\delta} \times B_{\delta}} \frac{\left|U_{\varepsilon}(x)-U_{\varepsilon}(y)\right|^{2}}{|x-y|^{n+2 s}} d x d y \\
& +2 \int_{\mathbb{D}} \frac{\left|U_{\varepsilon}(x)-U_{\varepsilon}(y)\right|^{2}}{|x-y|^{n+2 s}} d x d y+\mathcal{O}\left(\varepsilon^{n-2 s}\right) \\
\leqslant & \int_{\mathbb{R}^{2 n}} \frac{\left|U_{\varepsilon}(x)-U_{\varepsilon}(y)\right|^{2}}{|x-y|^{n+2 s}} d x d y+\mathcal{O}\left(\varepsilon^{n-2 s}\right),
\end{aligned}
$$

as $\varepsilon \rightarrow 0$. Then, the desired result now follows from Claim 7

Now we consider the $L^{2}$ and the $L^{2^{*}}$ norms of the function $u_{\varepsilon}$. In this case our estimates can be proved exactly as in the case of the Laplacian, but we prefer to repeat them, for the reader's convenience.

Proposition 22. Let $s \in(0,1)$ and $n>2 s$. Then, the following estimates hold true:

$$
\int_{\mathbb{R}^{n}}\left|u_{\varepsilon}(x)\right|^{2} d x \geqslant \begin{cases}C_{s} \varepsilon^{2 s}+\mathcal{O}\left(\varepsilon^{n-2 s}\right) & \text { if } n>4 s, \\ C_{s} \varepsilon^{2 s}|\log \varepsilon|+\mathcal{O}\left(\varepsilon^{2 s}\right) & \text { if } n=4 s, \\ C_{s} \varepsilon^{n-2 s}+\mathcal{O}\left(\varepsilon^{2 s}\right) & \text { if } n<4 s\end{cases}
$$

\footnotetext{
${ }^{5}$ It is interesting to observe that the energy interaction outside $B_{\delta} \times B_{\delta}$ is not negligible. Indeed, while the contributions in 4.24), 4.25), 4.28) and (4.31) are all $\mathcal{O}\left(\varepsilon^{n-2 s}\right)$, the integral in $\mathbb{D}$ provides a relevant part that needs to be taken into account in the full energy.
} 
and

$$
\int_{\mathbb{R}^{n}}\left|u_{\varepsilon}(x)\right|^{2^{*}} d x=S_{s}^{n /(2 s)}+\mathcal{O}\left(\varepsilon^{n}\right),
$$

as $\varepsilon \rightarrow 0$, for some positive constant $C_{s}$ depending on $s$.

Proof. By definition of $u_{\varepsilon}$ and (4.12) we get

$$
\begin{aligned}
\int_{\mathbb{R}^{n}}\left|u_{\varepsilon}(x)\right|^{2} d x & =\int_{B_{\delta}}\left|U_{\varepsilon}(x)\right|^{2} d x+\int_{B_{2 \delta} \backslash B_{\delta}}\left|\eta(x) U_{\varepsilon}(x)\right|^{2} d x \\
& \geqslant \varepsilon^{-(n-2 s)} \int_{B_{\delta}}\left|u^{*}(x / \varepsilon)\right|^{2} d x \\
& \geqslant \varepsilon^{2 s} \int_{R}^{\delta / \varepsilon}\left|u^{*}(r)\right|^{2} r^{n-1} d r
\end{aligned}
$$

for any $0<R<\delta / \varepsilon$.

Now, by (4.7) and Claim 5 we get

$$
\begin{aligned}
& \int_{\mathbb{R}^{n}}\left|u_{\varepsilon}(x)\right|^{2} d x \geqslant \varepsilon^{2 s} \int_{R}^{\delta / \varepsilon}\left|u^{*}(r)\right|^{2} r^{n-1} d r \\
& \cong \varepsilon^{2 s} \int_{R}^{\delta / \varepsilon} r^{-n+4 s-1} d r \\
& =\left\{\begin{array}{lll}
-C_{1, s} \varepsilon^{n-2 s}+C_{2, s} \varepsilon^{2 s} & \text { if } & n>4 s, \\
C_{1, s} \varepsilon^{2 s}|\log \varepsilon|+C_{2, s} \varepsilon^{2 s} & \text { if } & n=4 s, \\
C_{1, s} \varepsilon^{n-2 s}-C_{2, s} \varepsilon^{2 s} & \text { if } & n<4 s,
\end{array}\right. \\
& = \begin{cases}C_{s} \varepsilon^{2 s}+\mathcal{O}\left(\varepsilon^{n-2 s}\right) & \text { if } \quad n>4 s, \\
C_{s} \varepsilon^{2 s}|\log \varepsilon|+\mathcal{O}\left(\varepsilon^{2 s}\right) & \text { if } \quad n=4 s, \\
C_{s} \varepsilon^{n-2 s}+\mathcal{O}\left(\varepsilon^{2 s}\right) & \text { if } \quad n<4 s,\end{cases}
\end{aligned}
$$

as $\varepsilon \rightarrow 0$, for some positive constant $C_{1, s}, C_{2, s}$ and $C_{s}$, depending on $s$, and for $\varepsilon$ sufficiently small. This proves (4.32) and we now prove (4.33). Arguing in the same way and using Claim 7 , we have

$$
\begin{aligned}
\int_{\mathbb{R}^{n}}\left|u_{\varepsilon}(x)\right|^{2^{*}} d x & =\int_{\mathbb{R}^{n}}\left|U_{\varepsilon}(x)\right|^{2^{*}} d x+\int_{\mathbb{R}^{n}}\left(|\eta(x)|^{2^{*}}-1\right)\left|U_{\varepsilon}(x)\right|^{2^{*}} d x \\
& =S_{s}^{n /(2 s)}+\int_{\mathbb{R}^{n} \backslash B_{\delta}}\left(|\eta(x)|^{2^{*}}-1\right)\left|U_{\varepsilon}(x)\right|^{2^{*}} d x \\
& =S_{s}^{n /(2 s)}+\varepsilon^{-n} \int_{\mathbb{R}^{n} \backslash B_{\delta}}\left(|\eta(x)|^{2^{*}}-1\right)\left|u^{*}(x / \varepsilon)\right|^{2^{*}} d x \\
& \cong S_{s}^{n /(2 s)}+C \varepsilon^{n} \int_{\mathbb{R}^{n} \backslash B_{\delta}}|x|^{-2 n} d x \\
& =S_{s}^{n /(2 s)}+\mathcal{O}\left(\varepsilon^{n}\right)
\end{aligned}
$$

as $\varepsilon \rightarrow 0$. This ends the proof of Proposition 22 .

With Propositions 21 and 22 we can conclude the proof of Theorem 4. 
4.2.1. End of the proof of Theorem 4. We prove that $u_{\varepsilon}$ satisfies condition (4.2) if $\varepsilon$ is suitably small (recall also that $u_{\epsilon}$ vanishes outside $\Omega$ because of (4.15)). For this, let us consider two different cases.

Case 1: $n>4 s$. By the definition of the function $S_{s, \lambda}(\cdot)$ given in formula (1.24) and by Propositions 21 and 22 we get

$$
\begin{aligned}
S_{s, \lambda}\left(u_{\varepsilon}\right) & \leqslant \frac{S_{s}^{n /(2 s)}+\mathcal{O}\left(\varepsilon^{n-2 s}\right)-\lambda C_{s} \varepsilon^{2 s}}{\left(S_{s}^{n /(2 s)}+\mathcal{O}\left(\varepsilon^{n}\right)^{2 / 2^{*}}\right.} \\
& \leqslant S_{s}+\mathcal{O}\left(\varepsilon^{n-2 s}\right)-\lambda \widetilde{C}_{s} \varepsilon^{2 s} \\
& =S_{s}+\varepsilon^{2 s}\left(\mathcal{O}\left(\varepsilon^{n-4 s}\right)-\lambda \widetilde{C}_{s}\right)<S_{s},
\end{aligned}
$$

for any $\lambda>0$, if $\varepsilon>0$ is sufficiently small, for a suitable positive constant $\widetilde{C}_{s}$. Hence, condition (4.2) is satisfied (with $u_{0}=u_{\varepsilon}$ ) for any $\lambda>0$ when $n>4 s$. Thus, by Theorem 3 , for any $\lambda \in\left(0, \lambda_{1, s}\right)$ problem (1.30) admits a solution $u \in H^{s}\left(\mathbb{R}^{n}\right)$, which is not identically zero, such that $u=0$ a.e. in $\mathbb{R}^{n} \backslash \Omega$.

Case 2: $n=4 s$. Arguing as in Case 1 and taking into account that $n=4 s$ for some positive constant $\widetilde{C}_{s}$, we get

$$
\begin{aligned}
S_{s, \lambda}\left(u_{\varepsilon}\right) & \leqslant \frac{S_{s}^{n /(2 s)}+\mathcal{O}\left(\varepsilon^{n-2 s}\right)-\lambda C_{s} \varepsilon^{2 s}|\log \varepsilon|+\mathcal{O}\left(\varepsilon^{2 s}\right)}{\left(S_{s}^{n /(2 s)}+\mathcal{O}\left(\varepsilon^{n}\right)^{2 / 2^{*}}\right.} \\
& \leqslant S_{s}+\mathcal{O}\left(\varepsilon^{2 s}\right)-\lambda \widetilde{C}_{s} \varepsilon^{2 s}|\log \varepsilon| \\
& =S_{s}+\varepsilon^{2 s}\left(\mathcal{O}(1)-\lambda \widetilde{C}_{s}|\log \varepsilon|\right)<S_{s},
\end{aligned}
$$

for any $\lambda>0$, if $\varepsilon>0$ is sufficiently small. Hence, condition (4.2) is satisfied with $u_{0}=u_{\varepsilon}$ for any $\lambda>0$ when $n=4 s$. Therefore, thanks to Theorem 3 , for any $\lambda \in\left(0, \lambda_{1, s}\right)$ problem (1.30) admits a solution $u \in H^{s}\left(\mathbb{R}^{n}\right)$, which is not identically zero and such that $u=0$ a.e. in $\mathbb{R}^{n} \backslash \Omega$.

This completes the proof of Theorem 4 .

4.2.2. Some remarks on condition $S_{s, \lambda}<S_{s}$. As in the classical case (see the discussion in [3, pages 458-459]), and also in the non-local setting, the PalaisSmale condition may globally fail for the functional $\mathcal{J}_{s, \lambda}$. Therefore, it is necessary to restrict our attention only to a suitable range related to the best fractional critical Sobolev constant $S_{s}$ of the embedding $H^{s}\left(\mathbb{R}^{n}\right) \hookrightarrow L^{2^{*}}\left(\mathbb{R}^{n}\right)$. Indeed, this functional satisfies the Palais-Smale condition at any level $c$, with $c$ satisfying

$$
c<\frac{s}{n} S_{s}^{n /(2 s)} .
$$

As discussed in the introduction, another natural condition to look at is given by

$$
S_{s, \lambda}<S_{s} \text {. }
$$

It turns out that conditions (4.35) and (4.36) are related as follows:

Proposition 23. Let $0<\lambda<\lambda_{1, s}$, where $\lambda_{1, s}$ is the first eigenvalue of the operator $(-\Delta)^{s}$ with homogeneous Dirichlet boundary conditions and let

$$
c=\inf _{P \in \mathcal{P}} \sup _{v \in P([0,1])} \mathcal{J}_{s, \lambda}^{0}(v),
$$


where

$$
\mathcal{J}_{s, \lambda}^{0}(u)=\frac{1}{2} \int_{\mathbb{R}^{2 n}} \frac{|u(x)-u(y)|^{2}}{|x-y|^{n+2 s}} d x d y-\frac{\lambda}{2} \int_{\Omega}|u(x)|^{2} d x-\frac{1}{2^{*}} \int_{\Omega}|u(x)|^{2^{*}} d x,
$$

and

$$
\mathcal{P}=\left\{P \in C\left([0,1] ; X_{0}\right): P(0)=0, P(1)=e\right\}
$$

with $e \in H^{s}\left(\mathbb{R}^{n}\right)$ such that $\mathcal{J}_{s, \lambda}^{0}(e)<0$.

Then, conditions (4.35) and (4.36) are equivalent.

Proof. It is enough to show that

$$
c=\frac{s}{n} S_{s, \lambda}^{n /(2 s)} .
$$

For this, let $u \in H^{s}\left(\mathbb{R}^{n}\right) \backslash\{0\}$ and let $P(\zeta)=\zeta u$ with $\zeta \in[0,1]$. By (4.1) and (4.37) it is easily seen that

$$
c \leqslant \sup _{\zeta \geqslant 0} \mathcal{J}_{s, \lambda}(\zeta u)=\frac{s}{n} S_{s, \lambda}^{n /(2 s)}(u)
$$

so that, taking the infimum and using the definition (1.23), we obtain

$$
c \leqslant \frac{s}{n} S_{s, \lambda}^{n /(2 s)}
$$

Now, let us prove the other inequality. In order to do this let us consider the continuous function

$$
[0,1] \ni \zeta \mapsto\left\langle\left(\mathcal{J}_{s, \lambda}^{0}\right)^{\prime}(P(\zeta)), P(\zeta)\right\rangle,
$$

for any fixed $P \in \mathcal{P}$. Let

$$
\widetilde{\zeta}:=\sup \{\zeta \in[0,1]: P(\zeta)=0\} .
$$

Since $P(0)=0, P(1)=e \neq 0$ and $P$ is continuous, it follows that

$$
\widetilde{\zeta} \in[0,1) .
$$

Since $2<2^{*}$ and $\mathcal{J}_{s, \lambda}^{0}(e)<0$ by assumption, we have that

$$
\begin{aligned}
\left\langle\left(\mathcal{J}_{s, \lambda}^{0}\right)^{\prime}(e), e\right\rangle & =\int_{\mathbb{R}^{2 n}} \frac{|e(x)-e(y)|^{2}}{|x-y|^{n+2 s}} d x d y-\lambda \int_{\Omega}|e(x)|^{2} d x-\int_{\Omega}|e(x)|^{2^{*}} d x \\
& =2 \mathcal{J}_{s, \lambda}^{0}(e)+\left(\frac{2}{2^{*}}-1\right) \int_{\Omega}|e(x)|^{2^{*}} d x \\
& <2 \mathcal{J}_{s, \lambda}^{0}(e)<0 .
\end{aligned}
$$

On the other hand, since $0<\lambda<\lambda_{1, s}$, we get

$$
\begin{gathered}
\left\langle\left(\mathcal{J}_{s, \lambda}^{0}\right)^{\prime}(P(\zeta)), P(\zeta)\right\rangle=\int_{\mathbb{R}^{2 n}} \frac{|P(\zeta)(x)-P(\zeta)(y)|^{2}}{|x-y|^{n+2 s}} d x d y-\lambda \int_{\Omega}|P(\zeta)(x)|^{2} d x \\
-\int_{\Omega}|P(\zeta)(x)|^{2^{*}} d x \\
\geqslant\left(1-\frac{\lambda}{\lambda_{1, s}}\right) \int_{\mathbb{R}^{2 n}} \frac{|P(\zeta)(x)-P(\zeta)(y)|^{2}}{|x-y|^{n+2 s}} d x d y \\
-\|P(\zeta)\|_{L^{2^{*}}(\Omega)} .
\end{gathered}
$$


So, by Lemma 8 r $a$ ) and the fact that $2<2^{*}$,

$$
\left\langle\left(\mathcal{J}_{s, \lambda}^{0}\right)^{\prime}(P(\zeta)), P(\zeta)\right\rangle \geqslant \frac{1}{c}\left(1-\frac{\lambda}{\lambda_{1, s}}\right)\|P(\zeta)\|_{L^{2^{*}}(\Omega)}^{2}-\|P(\zeta)\|_{L^{2^{*}(\Omega)}}^{2^{*}}>0,
$$

provided that

$$
\|P(\zeta)\|_{L^{2^{*}(\Omega)}} \in\left(0,\left[\frac{1}{c}\left(1-\frac{\lambda}{\lambda_{1, s}}\right)\right]^{1 /\left(2^{*}-2\right)}\right) .
$$

In particular, since $P$ is continuous, recalling (4.40) and (4.41), we obtain that there exists $\widehat{\zeta} \in(\widetilde{\zeta}, 1)$ such that

$$
\left\langle\left(\mathcal{J}_{s, \lambda}^{0}\right)^{\prime}(P(\zeta)), P(\zeta)\right\rangle>0 \text { for any } \zeta \in(\widetilde{\zeta}, \widehat{\zeta}) .
$$

As a consequence of (4.42) and (4.43) we get that there exists $\bar{\zeta} \in(\widehat{\zeta}, 1)$ such that $\bar{u}:=P(\bar{\zeta})$ satisfies

$$
\begin{aligned}
\left\langle\left(\mathcal{J}_{s, \lambda}^{0}\right)^{\prime}(\bar{u}), \bar{u}\right\rangle= & \int_{\mathbb{R}^{2 n}} \frac{|\bar{u}(x)-\bar{u}(y)|^{2}}{|x-y|^{n+2 s}} d x d y \\
& \quad-\lambda \int_{\Omega}|\bar{u}(x)|^{2} d x-\int_{\Omega}|\bar{u}(x)|^{2^{*}} d x=0 .
\end{aligned}
$$

We stress that

$$
\bar{u} \neq 0 \text {, }
$$

because $\bar{\zeta}>\widehat{\zeta}>\widetilde{\zeta}$ by construction, and so $P(\bar{\zeta}) \neq 0$ by (4.40).

Also, using the definition of $\mathcal{J}_{s, \lambda}^{0}$ and (4.44) we easily get

$$
\mathcal{J}_{s, \lambda}^{0}(\bar{u})=\left(\frac{1}{2}-\frac{1}{2^{*}}\right)\|\bar{u}\|_{L^{2^{*}}}^{2^{*}}=\frac{s}{n}\|\bar{u}\|_{L^{2^{*}}}^{2^{*}}
$$

Hence, by (1.23), it holds true that

$$
\begin{aligned}
S_{s, \lambda} & \leqslant S_{s, \lambda}(\bar{u})=\frac{\int_{\mathbb{R}^{2 n}} \frac{|\bar{u}(x)-\bar{u}(y)|^{2}}{|x-y|^{n+2 s}} d x d y-\lambda \int_{\mathbb{R}^{n}}|\bar{u}(x)|^{2} d x}{\left(\int_{\mathbb{R}^{n}}|\bar{u}(x)|^{2^{*}} d x\right)^{2 / 2^{*}}} \\
& \leqslant \frac{\int_{\mathbb{R}^{2 n}} \frac{|\bar{u}(x)-\bar{u}(y)|^{2}}{|x-y|^{n+2 s}} d x d y-\lambda \int_{\Omega}|\bar{u}(x)|^{2} d x}{\left(\int_{\Omega}|\bar{u}(x)|^{2^{*}} d x\right)^{2 / 2^{*}}} \\
& =\left(\int_{\Omega}|\bar{u}(x)|^{2^{*}} d x\right)^{\left(2^{*}-2\right) / 2^{*}}=\left(\frac{n}{s} \mathcal{J}_{s, \lambda}^{0}(\bar{u})\right)^{2 s / n} \\
& \leqslant\left(\frac{n}{s} \sup _{v \in P([0,1])} \mathcal{J}_{s, \lambda}^{0}(v)\right)^{2 s / n},
\end{aligned}
$$

thanks to (4.44) - (4.46). Taking the infimum in $P \in \mathcal{P}$, we conclude that

$$
S_{s, \lambda} \leqslant\left(\frac{n}{s} c\right)^{2 s / n}
$$

that is

$$
c \geqslant \frac{s}{n} S_{s, \lambda}^{n /(2 s)} .
$$

By (4.39) and (4.47) we deduce (4.38), and this ends the proof of Proposition 23 , 


\section{REFERENCES}

[1] Antonio Ambrosetti and Paul H. Rabinowitz, Dual variational methods in critical point theory and applications, J. Functional Analysis 14 (1973), 349-381. MR0370183 (51 \#6412)

[2] Haïm Brezis, Analyse fonctionnelle (French), Collection Mathématiques Appliquées pour la Maîtrise. [Collection of Applied Mathematics for the Master's Degree], Masson, Paris, 1983. Théorie et applications. [Theory and applications]. MR697382 (85a:46001)

[3] Haïm Brézis and Louis Nirenberg, Positive solutions of nonlinear elliptic equations involving critical Sobolev exponents, Comm. Pure Appl. Math. 36 (1983), no. 4, 437-477, DOI 10.1002/cpa.3160360405. MR709644 (84h:35059)

[4] Luis Caffarelli and Luis Silvestre, An extension problem related to the fractional Laplacian, Comm. Partial Differential Equations 32 (2007), no. 7-9, 1245-1260, DOI 10.1080/03605300600987306. MR.2354493 (2009k:35096)

[5] A. Capozzi, D. Fortunato, and G. Palmieri, An existence result for nonlinear elliptic problems involving critical Sobolev exponent, Ann. Inst. H. Poincaré Anal. Non Linéaire 2 (1985), no. 6, 463-470. MR831041 (87j:35126)

[6] Athanase Cotsiolis and Nikolaos K. Tavoularis, Best constants for Sobolev inequalities for higher order fractional derivatives, J. Math. Anal. Appl. 295 (2004), no. 1, 225-236, DOI 10.1016/j.jmaa.2004.03.034. MR2064421 (2005d:46070)

[7] A. Fiscella, R. Servadei, and E. Valdinoci, Density properties for fractional Sobolev spaces, to appear in Ann. Acad. Sci. Fenn. Math.

[8] Rafael de la Llave and Enrico Valdinoci, Symmetry for a Dirichlet-Neumann problem arising in water waves, Math. Res. Lett. 16 (2009), no. 5, 909-918. MR2576707 (2011b:35350)

[9] Eleonora Di Nezza, Giampiero Palatucci, and Enrico Valdinoci, Hitchhiker's guide to the fractional Sobolev spaces, Bull. Sci. Math. 136 (2012), no. 5, 521-573, DOI 10.1016/j.bulsci.2011.12.004. MR2944369

[10] Paul H. Rabinowitz, Some critical point theorems and applications to semilinear elliptic partial differential equations, Ann. Scuola Norm. Sup. Pisa Cl. Sci. (4) 5 (1978), no. 1, 215223. MR0488128 (58 \#7695)

[11] Paul H. Rabinowitz, Minimax methods in critical point theory with applications to differential equations, CBMS Regional Conference Series in Mathematics, vol. 65, published for the Conference Board of the Mathematical Sciences, Washington, DC, 1986. MR 845785 (87j:58024)

[12] Raffaella Servadei and Enrico Valdinoci, A multiplicity result for a class of nonlinear variational inequalities, Nonlinear Stud. 12 (2005), no. 1, 37-48. MR2116874 (2005k:35162)

[13] R. Servadei and E. Valdinoci, Lewy-Stampacchia type estimates for variational inequalities driven by (non)local operators, Rev. Mat. Iberoam. 29 (2013), no. 3, 1091-1126. MR3090147

[14] Raffaella Servadei and Enrico Valdinoci, Mountain pass solutions for non-local elliptic operators, J. Math. Anal. Appl. 389 (2012), no. 2, 887-898, DOI 10.1016/j.jmaa.2011.12.032. MR2879266 (2012k:35095)

[15] R. Servadei and E. Valdinoci, Variational methods for non-local operators of elliptic type, Discrete Contin. Dyn. Syst. 33 (2013), no. 5, 2105-2137. MR.3002745

[16] Michael Struwe, Variational methods, Springer-Verlag, Berlin, 1990. Applications to nonlinear partial differential equations and Hamiltonian systems. MR1078018 (92b:49002)

[17] Jinggang Tan, The Brezis-Nirenberg type problem involving the square root of the Laplacian, Calc. Var. Partial Differential Equations 42 (2011), no. 1-2, 21-41, DOI 10.1007/s00526-0100378-3. MR2819627 (2012e:35079)

[18] Michel Willem, Minimax theorems, Progress in Nonlinear Differential Equations and their Applications, 24, Birkhäuser Boston Inc., Boston, MA, 1996. MR1400007 (97h:58037)

Dipartimento di Matematica e Informatica, Università della Calabria, Ponte Pietro Bucci 31 B, 87036 Arcavacata di Rende, Cosenza, Italy

E-mail address: servadei@mat.unical.it

Dipartimento di Matematica, Università di Milano, Via Cesare Saldini 50, 20133 MiLano, Italy and Weierstrass Institut für Angewandte Analysis und Stochastik, MohrenStrasse 39, D-10117 Berlin, Germany and Istituto di Matematica Applicata e Tecnologie Informatiche, Consiglio Nazionale delle Ricerche, Via Ferrata 1, 27100 Pavia, Italy

E-mail address: enrico.valdinoci@unimi.it 